\title{
COMPATIBLE FLAT METRICS
}

\section{OLEG I. MOKHOV}

Received 13 December 2001

We solve the problem of description of nonsingular pairs of compatible flat metrics for the general $N$-component case. The integrable nonlinear partial differential equations describing all nonsingular pairs of compatible flat metrics (or, in other words, nonsingular flat pencils of metrics) are found and integrated. The integrating of these equations is based on reducing to a special nonlinear differential reduction of the Lamé equations and using the Zakharov method of differential reductions in the dressing method (a version of the inverse scattering method).

\section{Introduction and basic definitions}

We use both contravariant metrics $g^{i j}(u)$ with upper indices, where $u=$ $\left(u^{1}, \ldots, u^{N}\right)$ are local coordinates, $1 \leq i, j \leq N$, and covariant metrics $g_{i j}(u)$ with lower indices, $g^{i s}(u) g_{s j}(u)=\delta_{j}^{i}$. Indices of coefficients of the LeviCivita connections $\Gamma_{j k}^{i}(u)$ and indices of the tensors of Riemannian curvature $R_{j k l}^{i}(u)$ are raised and lowered by the metrics corresponding to them

$$
\begin{aligned}
\Gamma_{k}^{i j}(u) & =g^{i s}(u) \Gamma_{s k}^{j}(u), \\
\Gamma_{j k}^{i}(u) & =\frac{1}{2} g^{i s}(u)\left(\frac{\partial g_{s k}}{\partial u^{j}}+\frac{\partial g_{j s}}{\partial u^{k}}-\frac{\partial g_{j k}}{\partial u^{s}}\right), \\
R_{k l}^{i j}(u) & =g^{i s}(u) R_{s k l}^{j}(u), \\
R_{j k l}^{i}(u) & =\frac{\partial \Gamma_{j l}^{i}}{\partial u^{k}}-\frac{\partial \Gamma_{j k}^{i}}{\partial u^{l}}+\Gamma_{p k}^{i}(u) \Gamma_{j l}^{p}(u)-\Gamma_{p l}^{i}(u) \Gamma_{j k}^{p}(u) .
\end{aligned}
$$


Definition 1.1. Two contravariant flat metrics $g_{1}^{i j}(u)$ and $g_{2}^{i j}(u)$ are called compatible if any linear combination of these metrics

$$
g^{i j}(u)=\lambda_{1} g_{1}^{i j}(u)+\lambda_{2} g_{2}^{i j}(u),
$$

where $\lambda_{1}$ and $\lambda_{2}$ are arbitrary constants such that $\operatorname{det}\left(g^{i j}(u)\right) \not \equiv 0$, is also a flat metric and coefficients of the corresponding Levi-Civita connections are related by the same linear formula

$$
\Gamma_{k}^{i j}(u)=\lambda_{1} \Gamma_{1, k}^{i j}(u)+\lambda_{2} \Gamma_{2, k}^{i j}(u) .
$$

We also say in this case that the flat metrics $g_{1}^{i j}(u)$ and $g_{2}^{i j}(u)$ form a flat pencil (this definition was proposed by Dubrovin in $[6,7]$ ).

Definition 1.2. Two contravariant metrics $g_{1}^{i j}(u)$ and $g_{2}^{i j}(u)$ of constant Riemannian curvature $K_{1}$ and $K_{2}$, respectively, are called compatible if any linear combination of these metrics

$$
g^{i j}(u)=\lambda_{1} g_{1}^{i j}(u)+\lambda_{2} g_{2}^{i j}(u),
$$

where $\lambda_{1}$ and $\lambda_{2}$ are arbitrary constants such that $\operatorname{det}\left(g^{i j}(u)\right) \neq \equiv$, is a metric of constant Riemannian curvature $\lambda_{1} K_{1}+\lambda_{2} K_{2}$, and coefficients of the corresponding Levi-Civita connections are related by the same linear formula

$$
\Gamma_{k}^{i j}(u)=\lambda_{1} \Gamma_{1, k}^{i j}(u)+\lambda_{2} \Gamma_{2, k}^{i j}(u) .
$$

We also say in this case that the metrics $g_{1}^{i j}(u)$ and $g_{2}^{i j}(u)$ form a pencil of metrics of constant Riemannian curvature.

Definition 1.3. Two Riemannian or pseudo-Riemannian contravariant metrics $g_{1}^{i j}(u)$ and $g_{2}^{i j}(u)$ are called compatible if, for any linear combination of these metrics

$$
g^{i j}(u)=\lambda_{1} g_{1}^{i j}(u)+\lambda_{2} g_{2}^{i j}(u),
$$

where $\lambda_{1}$ and $\lambda_{2}$ are arbitrary constants such that $\operatorname{det}\left(g^{i j}(u)\right) \not \equiv 0$, coefficients of the corresponding Levi-Civita connections and components of the corresponding tensors of Riemannian curvature are related by the same linear formula

$$
\begin{aligned}
\Gamma_{k}^{i j}(u) & =\lambda_{1} \Gamma_{1, k}^{i j}(u)+\lambda_{2} \Gamma_{2, k}^{i j}(u), \\
R_{k l}^{i j}(u) & =\lambda_{1} R_{1, k l}^{i j}(u)+\lambda_{2} R_{2, k l}^{i j}(u) .
\end{aligned}
$$


We also say in this case that the metrics $g_{1}^{i j}(u)$ and $g_{2}^{i j}(u)$ form a pencil of metrics.

Definition 1.4. Two Riemannian or pseudo-Riemannian contravariant metrics $g_{1}^{i j}(u)$ and $g_{2}^{i j}(u)$ are called almost compatible if, for any linear combination of the metrics (1.6), relation (1.7) is fulfilled.

Definition 1.5. Two Riemannian or pseudo-Riemannian metrics $g_{1}^{i j}(u)$ and $g_{2}^{i j}(u)$ are called a nonsingular pair of metrics if the eigenvalues of this pair of metrics, that is, roots of the equation

$$
\operatorname{det}\left(g_{1}^{i j}(u)-\lambda g_{2}^{i j}(u)\right)=0,
$$

are distinct.

A pencil of metrics is called nonsingular if it is formed by a nonsingular pair of metrics.

These definitions are motivated by the theory of compatible Poisson brackets of hydrodynamic type. We give a brief survey of this theory in the next section. In the case if the metrics $g_{1}^{i j}(u)$ and $g_{2}^{i j}(u)$ are flat, that is, $R_{1, j k l}^{i}(u)=R_{2, j k l}^{i}(u)=0$, relation (1.8) is equivalent to the condition that an arbitrary linear combination of the flat metrics $g_{1}^{i j}(u)$ and $g_{2}^{i j}(u)$ is also a flat metric. In this case, Definition 1.3 is equivalent to the well-known definition of a flat pencil of metrics (Definition 1.1) or, in other words, a compatible pair of local nondegenerate Poisson structures of hydrodynamic type [6] (see also $[7,8,13,25,26,27,28,29]$ ). In the case if the metrics $g_{1}^{i j}(u)$ and $g_{2}^{i j}(u)$ are metrics of constant Riemannian curvature $K_{1}$ and $K_{2}$, respectively, that is,

$$
R_{1, k l}^{i j}(u)=K_{1}\left(\delta_{l}^{i} \delta_{k}^{j}-\delta_{k}^{i} \delta_{l}^{j}\right), \quad R_{2, k l}^{i j}(u)=K_{2}\left(\delta_{l}^{i} \delta_{k}^{j}-\delta_{k}^{i} \delta_{l}^{j}\right),
$$

relation (1.8) gives the condition that an arbitrary linear combination of the metrics $g_{1}^{i j}(u)$ and $g_{2}^{i j}(u),(1.6)$, is a metric of constant Riemannian curvature $\lambda_{1} K_{1}+\lambda_{2} K_{2}$. In this case, Definition 1.3 is equivalent to our Definition 1.2 of a pencil of metrics of constant Riemannian curvature or, in other words, a compatible pair of the corresponding nonlocal Poisson structures of hydrodynamic type, which were introduced and studied by the author and Ferapontov in [30]. Compatible metrics of more general type correspond to a compatible pair of nonlocal Poisson structures of hydrodynamic type that were introduced and studied by Ferapontov in [12]. They arise, for example, if we use a recursion operator generated by a pair of compatible Poisson structures of hydrodynamic type. Such 
recursion operators determine, as is well known, infinite sequences of corresponding (generally speaking, nonlocal) Poisson structures.

As noted earlier by the author in $[26,27,28,29]$, condition (1.8) follows from condition (1.7) in the case of certain special reductions connected with the associativity equations (see also Theorem 3.5 below). Of course, it is not by chance. Under certain very natural and quite general assumptions on metrics (it is sufficient but not necessary, in particular, that eigenvalues of the pair of metrics under consideration are distinct), compatibility of the metrics follows from their almost compatibility, but, generally speaking, in the general case, it is not true even for flat metrics (we will present the corresponding counterexamples below). Correspondingly, we would like to emphasize that condition (1.7), which is considerably more simple than condition (1.8), almost guarantees compatibility of metrics and deserves a separate study, but, in the general case, it is also necessary to require the fulfillment of condition (1.8) for compatibility of the corresponding Poisson structures of hydrodynamic type. It is also interesting to find out whether condition (1.8) guarantees the fulfillment of condition (1.7).

This paper is devoted to the problem of description of all nonsingular pairs of compatible flat metrics and to integrability of the corresponding nonlinear partial differential equations by the inverse scattering method.

\section{Compatible local Poisson structures of hydrodynamic type}

Any local homogeneous first-order Poisson bracket, that is, a Poisson bracket of the form

$$
\left\{u^{i}(x), u^{j}(y)\right\}=g^{i j}(u(x)) \delta_{x}(x-y)+b_{k}^{i j}(u(x)) u_{x}^{k} \delta(x-y),
$$

where $u^{1}, \ldots, u^{N}$ are local coordinates on a certain smooth $N$-dimensional manifold $M$, is called a local Poisson structure of hydrodynamic type or Dubrovin-Novikov structure [9]. Here, $u^{i}(x), 1 \leq i \leq N$, are functions (fields) of a single independent variable $x$, and coefficients $g^{i j}(u)$ and $b_{k}^{i j}(u)$ of bracket (2.1) are smooth functions of local coordinates.

In other words, for arbitrary functionals $I[u]$ and $J[u]$ on the space of fields $u^{i}(x), 1 \leq i \leq N$, a bracket of the form

$$
\{I, J\}=\int \frac{\delta I}{\delta u^{i}(x)}\left(g^{i j}(u(x)) \frac{d}{d x}+b_{k}^{i j}(u(x)) u_{x}^{k}\right) \frac{\delta J}{\delta u^{j}(x)} d x
$$

is defined, and it is required that this bracket is a Poisson bracket, that is, it is skew-symmetric

$$
\{I, J\}=-\{J, I\}
$$


and satisfies the Jacobi identity

$$
\{\{I, J\}, K\}+\{\{J, K\}, I\}+\{\{K, I\}, J\}=0
$$

for arbitrary functionals $I[u], J[u]$, and $K[u]$. The skew-symmetry (2.3) and the Jacobi identity (2.4) impose very restrictive conditions on coefficients $g^{i j}(u)$ and $b_{k}^{i j}(u)$ of bracket (2.2) (these conditions will be considered below). For bracket (2.2), the Leibniz identity

$$
\{I J, K\}=I\{J, K\}+J\{I, K\}
$$

is automatically fulfilled in accordance with the following property of variational derivative of functionals

$$
\frac{\delta(I J)}{\delta u^{i}(x)}=I \frac{\delta J}{\delta u^{i}(x)}+J \frac{\delta I}{\delta u^{i}(x)} .
$$

Recall that variational derivative of an arbitrary functional $I[u]$ is defined by

$$
\delta I \equiv I[u+\delta u]-I[u]=\int \frac{\delta I}{\delta u^{k}(x)} \delta u^{k}(x) d x+o(\delta u) .
$$

The definition of a local Poisson structure of hydrodynamic type does not depend on a choice of local coordinates $u^{1}, \ldots, u^{N}$ on the manifold $M$. Actually, the form of brackets (2.2) is invariant under local changes of coordinates $u^{i}=u^{i}\left(v^{1}, \ldots, v^{N}\right), 1 \leq i \leq N$, on $M$

$$
\begin{aligned}
& \int \frac{\delta I}{\delta u^{i}(x)}\left(g^{i j}(u(x)) \frac{d}{d x}+b_{k}^{i j}(u(x)) u_{x}^{k}\right) \frac{\delta J}{\delta u^{j}(x)} d x \\
& =\int \frac{\delta I}{\delta v^{i}(x)}\left(\tilde{g}^{i j}(v(x)) \frac{d}{d x}+\tilde{b}_{k}^{i j}(v(x)) v_{x}^{k}\right) \frac{\delta J}{\delta v^{j}(x)} d x,
\end{aligned}
$$

since variational derivatives of functionals transform like covector fields

$$
\frac{\delta I}{\delta v^{i}(x)}=\frac{\delta I}{\delta u^{s}(x)} \frac{\partial u^{s}}{\partial v^{i}} \text {. }
$$

Correspondingly, coefficients $g^{i j}(u)$ and $b_{k}^{i j}(u)$ of bracket (2.2) transform as follows:

$$
\begin{gathered}
\tilde{g}^{s r}(v)=g^{i j}(u(v)) \frac{\partial v^{s}}{\partial u^{i}} \frac{\partial v^{r}}{\partial u^{j}}, \\
\widetilde{b}_{l}^{s r}(v)=b_{k}^{i j}(u(v)) \frac{\partial v^{s}}{\partial u^{i}} \frac{\partial v^{r}}{\partial u^{j}} \frac{\partial u^{k}}{\partial v^{l}}+g^{i j}(u(v)) \frac{\partial v^{s}}{\partial u^{i}} \frac{\partial^{2} v^{r}}{\partial u^{j} \partial u^{p}} \frac{\partial u^{p}}{\partial v^{l}} .
\end{gathered}
$$


In particular, the coefficients $g^{i j}(u)$ define a contravariant tensor field of rank 2 (a contravariant "metric") on the manifold $M$. For the important case of a nondegenerate metric $g^{i j}(u), \operatorname{det} g^{i j} \neq 0$, (i.e., in the case of a pseudo-Riemannian manifold $\left.\left(M, g^{i j}\right)\right)$, the coefficients $b_{k}^{i j}(u)$ define the Christoffel symbols of an affine connection $\Gamma_{j k}^{i}(u)$ as follows:

$$
\begin{gathered}
b_{k}^{i j}(u)=-g^{i s}(u) \Gamma_{s k}^{j}(u), \\
\widetilde{\Gamma}_{j k}^{i}(v)=\Gamma_{r s}^{p}(u(v)) \frac{\partial v^{i}}{\partial u^{p}} \frac{\partial u^{r}}{\partial v^{j}} \frac{\partial u^{s}}{\partial v^{k}}+\frac{\partial^{2} u^{s}}{\partial v^{j} \partial v^{k}} \frac{\partial v^{i}}{\partial u^{s}} .
\end{gathered}
$$

The local Poisson structures of hydrodynamic type (2.1) were introduced and studied by Dubrovin and Novikov in [9], where they proposed a general local Hamiltonian approach (this approach corresponds to the local structures of the form (2.1)) to the so-called homogeneous systems of hydrodynamic type, that is, evolutionary quasilinear systems of first-order partial differential equations

$$
u_{t}^{i}=V_{j}^{i}(u) u_{x}^{j} .
$$

This Hamiltonian approach was motivated by the study of the equations of Euler hydrodynamics and the Whitham averaging equations, which describe the evolution of slowly modulated multiphase solutions of partial differential equations [10].

Local bracket $(2.2)$ is called nondegenerate if $\operatorname{det}\left(g^{i j}(u)\right) \not \equiv 0$. For the general nondegenerate brackets of form (2.2), Dubrovin and Novikov proved the following important theorem.

Theorem 2.1 (Dubrovin and Novikov [9]). If $\operatorname{det}\left(g^{i j}(u)\right) \equiv \equiv$, then bracket (2.2) is a Poisson bracket, that is, it is skew-symmetric and satisfies the Jacobi identity, if and only if

(1) $g^{i j}(u)$ is an arbitrary flat pseudo-Riemannian contravariant metric (a metric of zero Riemannian curvature),

(2) $b_{k}^{i j}(u)=-g^{i s}(u) \Gamma_{s k}^{j}(u)$, where $\Gamma_{s k}^{j}(u)$ is the Riemannian connection generated by the contravariant metric $g^{i j}(u)$ (the Levi-Civita connection).

Consequently, for any local nondegenerate Poisson structure of hydrodynamic type, there always exist local coordinates $v^{1}, \ldots, v^{N}$ (flat coordinates of the metric $\left.g^{i j}(u)\right)$ in which all coefficients of the bracket are constant:

$$
\tilde{g}^{i j}(v)=\eta^{i j}=\text { const }, \quad \tilde{\Gamma}_{j k}^{i}(v)=0, \quad \tilde{b}_{k}^{i j}(v)=0,
$$


that is, the bracket has the constant form

$$
\{I, J\}=\int \frac{\delta I}{\delta v^{i}(x)} \eta^{i j} \frac{d}{d x} \frac{\delta J}{\delta v^{j}(x)} d x
$$

where $\left(\eta^{i j}\right)$ is a nondegenerate symmetric constant matrix

$$
\eta^{i j}=\eta^{j i}, \quad \eta^{i j}=\text { const }, \quad \operatorname{det}\left(\eta^{i j}\right) \neq 0 .
$$

On the other hand, as early as 1978, Magri proposed a bi-Hamiltonian approach to the integration of nonlinear systems [22]. This approach demonstrated that integrability is closely related to the bi-Hamiltonian property, that is, to the property of a system to have two compatible Hamiltonian representations. As shown by Magri in [22], compatible Poisson brackets generate integrable hierarchies of systems of differential equations. Therefore, the description of compatible Poisson structures is very urgent and important problem in the theory of integrable systems. For a system, the bi-Hamiltonian property, in particular, generates recurrent relations for the conservation laws of this system.

Beginning from [22], quite extensive literature (see, e.g., [5, 15, 16, $18,34]$, and the necessary references therein) has been devoted to the bi-Hamiltonian approach and to the construction of compatible Poisson structures for many specific important equations of mathematical physics and field theory. Apparently, as far as the problem of description of sufficiently wide classes of compatible Poisson structures of defined special types is concerned, the first such statement was considered in [23, 24] (see also [2, 3]). In those papers, the author posed and completely solved the problem of description of all compatible local scalar first- and third-order Poisson brackets, that is, all Poisson brackets given by arbitrary scalar first- and third-order ordinary differential operators. These brackets generalize the well-known compatible pair of the GardnerZakharov-Faddeev bracket [17, 37] (the first-order bracket) and the Magri bracket [22] (the third-order bracket) for the Korteweg-de Vries equation.

In the case of homogeneous systems of hydrodynamic type, many integrable systems possess compatible Poisson structures of hydrodynamic type. The problems of description of these structures for particular systems and numerous examples were considered in many papers (see, e.g., [1, 14, 19, 31, 33, 35]). In [33] in particular, Nutku studied a special class of compatible two-component Poisson structures of hydrodynamic type and the related bi-Hamiltonian hydrodynamic systems. 
In [11], Ferapontov classified all two-component homogeneous systems of hydrodynamic type possessing three compatible nondegenerate local Poisson structures of hydrodynamic type.

In the general form, the problem of description of flat pencils of metrics (or, in other words, compatible nondegenerate local Poisson structures of hydrodynamic type) was considered by Dubrovin in $[6,7]$ in connection with the construction of important examples of such flat pencils of metrics generated by natural pairs of flat metrics on the spaces of orbits of Coxeter groups and on other Frobenius manifolds and associated with the corresponding quasi-homogeneous solutions of the associativity equations. In the theory of Frobenius manifolds introduced and studied by Dubrovin [6, 7] (they correspond to two-dimensional topological field theories), a key role is played by flat pencils of metrics, possessing a number of special additional (and very restrictive) properties (they satisfy the so-called quasi-homogeneity property). In addition, Dubrovin proved in [8] that the theory of Frobenius manifolds is equivalent to the theory of quasi-homogeneous compatible nondegenerate local Poisson structures of hydrodynamic type. The general problem on compatible nondegenerate local Poisson structures of hydrodynamic type was also considered by Ferapontov in [13].

The present author devoted $[25,26,27,28,29]$ to the general problem of classification of all compatible local Poisson structures of hydrodynamic type and to the study of the integrable nonlinear systems that describe the compatible Poisson structures and, mainly, the special reductions connected with the associativity equations.

Definition 2.2 (Magri [22]). Two Poisson brackets $\{,\}_{1}$ and $\{,\}_{2}$ are called compatible if an arbitrary linear combination of these Poisson brackets

$$
\{,\}=\lambda_{1}\{,\}_{1}+\lambda_{2}\{,\}_{2},
$$

where $\lambda_{1}$ and $\lambda_{2}$ are arbitrary constants, is also a Poisson bracket. In this case, we also say that the brackets $\{,\}_{1}$ and $\{,\}_{2}$ form a pencil of Poisson brackets.

Correspondingly, the problem of description of compatible nondegenerate local Poisson structures of hydrodynamic type is pure differentialgeometric problem of description of flat pencils of metrics (see [6, 7]).

In $[6,7]$, Dubrovin presented all the tensor relations for the general flat pencils of metrics. First, we introduce the necessary notation. Let $\nabla_{1}$ and $\nabla_{2}$ be the operators of covariant differentiation given by the LeviCivita connections $\Gamma_{1, k}^{i j}(u)$ and $\Gamma_{2, k}^{i j}(u)$ generated by the metrics $g_{1}^{i j}(u)$ 
and $g_{2}^{i j}(u)$, respectively. Indices of the covariant differentials are raised and lowered by the corresponding metrics

$$
\nabla_{1}^{i}=g_{1}^{i s}(u) \nabla_{1, s}, \quad \nabla_{2}^{i}=g_{2}^{i s}(u) \nabla_{2, s}
$$

Consider the tensor

$$
\Delta^{i j k}(u)=g_{1}^{i s}(u) g_{2}^{j p}(u)\left(\Gamma_{2, p s}^{k}(u)-\Gamma_{1, p s}^{k}(u)\right)
$$

introduced by Dubrovin in [6, 7].

THEOREM 2.3 (Dubrovin $[6,7]$ ). If metrics $g_{1}^{i j}(u)$ and $g_{2}^{i j}(u)$ form a flat pencil, then there exists a vector field $f^{i}(u)$ such that the tensor $\Delta^{i j k}(u)$ and the metric $g_{1}^{i j}(u)$ have the form

$$
\begin{gathered}
\Delta^{i j k}(u)=\nabla_{2}^{i} \nabla_{2}^{j} f^{k}(u), \\
g_{1}^{i j}(u)=\nabla_{2}^{i} f^{j}(u)+\nabla_{2}^{j} f^{i}(u)+c g_{2}^{i j}(u),
\end{gathered}
$$

where $c$ is a certain constant, and the vector field $f^{i}(u)$ satisfies the equations

$$
\Delta_{s}^{i j}(u) \Delta_{l}^{s k}(u)=\Delta_{s}^{i k}(u) \Delta_{l}^{s j}(u)
$$

where

$$
\begin{gathered}
\Delta_{k}^{i j}(u)=g_{2, k s}(u) \Delta^{s i j}(u)=\nabla_{2, k} \nabla_{2}^{i} f^{j}(u), \\
\left(g_{1}^{i s}(u) g_{2}^{j p}(u)-g_{2}^{i s}(u) g_{1}^{j p}(u)\right) \nabla_{2, s} \nabla_{2, p} f^{k}(u)=0 .
\end{gathered}
$$

Conversely, for the flat metric $g_{2}^{i j}(u)$ and the vector field $f^{i}(u)$ that is a solution of the system of (2.21) and (2.23), the metrics $g_{2}^{i j}(u)$ and (2.20) form a flat pencil.

The proof of this theorem immediately follows from the relations that are equivalent to the fact that the metrics $g_{1}^{i j}(u)$ and $g_{2}^{i j}(u)$ form a flat pencil and are considered in flat coordinates of the metric $g_{2}^{i j}(u)[6,7]$.

In [25], an explicit and simple criterion of compatibility for two local Poisson structures of hydrodynamic type is formulated; that is, it is shown what explicit form is sufficient and necessary for the local Poisson structures of hydrodynamic type to be compatible.

For the moment, in the general case, we are able to formulate such an explicit criterion only, namely, in terms of Poisson structures but not in terms of metrics as in Theorem 2.3. But for nonsingular pairs of the 
Poisson structures of hydrodynamic type (i.e., for nonsingular pairs of the corresponding metrics), we will, in this paper, get an explicit general criterion of compatibility, namely, in terms of the corresponding metrics.

Lemma 2.4 (an explicit criterion of compatibility for local Poisson structures of hydrodynamic type, [25]). Any local Poisson structure of hydrodynamic type $\{I, J\}_{2}$ is compatible with the constant nondegenerate Poisson bracket (2.14) if and only if it has the form

$$
\{I, J\}_{2}=\int \frac{\delta I}{\delta v^{i}(x)}\left(\left(\eta^{i s} \frac{\partial h^{j}}{\partial v^{s}}+\eta^{j s} \frac{\partial h^{i}}{\partial v^{s}}\right) \frac{d}{d x}+\eta^{i s} \frac{\partial^{2} h^{j}}{\partial v^{s} \partial v^{k}} v_{x}^{k}\right) \frac{\delta J}{\delta v^{j}(x)} d x,
$$

where $h^{i}(v), 1 \leq i \leq N$, are smooth functions defined on a certain neighbourhood.

We do not require in Lemma 2.4 that the Poisson structure of hydrodynamic type $\{I, J\}_{2}$ is nondegenerate. Besides, it is important to note that this statement is local.

In 1995, Ferapontov proposed in [13] an approach to the problem of flat pencils of metrics, which is motivated by the theory of recursion operators and formulated (without any proof) the following theorem as a criterion of compatibility for nondegenerate local Poisson structures of hydrodynamic type.

Theorem 2.5 [13]. Two local nondegenerate Poisson structures of hydrodynamic type given by flat metrics $g_{1}^{i j}(u)$ and $g_{2}^{i j}(u)$ are compatible if and only if the Nijenhuis tensor of the affinor $v_{j}^{i}(u)=g_{1}^{i s}(u) g_{2, s j}(u)$ vanishes, that is,

$$
N_{i j}^{k}(u)=v_{i}^{s}(u) \frac{\partial v_{j}^{k}}{\partial u^{s}}-v_{j}^{s}(u) \frac{\partial v_{i}^{k}}{\partial u^{s}}+v_{s}^{k}(u) \frac{\partial v_{i}^{s}}{\partial u^{j}}-v_{s}^{k}(u) \frac{\partial v_{j}^{s}}{\partial u^{i}}=0
$$

Besides, in the remark in [13], it is noted that if the spectrum of $v_{j}^{i}(u)$ is simple, then the vanishing of the Nijenhuis tensor implies the existence of coordinates $R^{1}, \ldots, R^{N}$ for which all the objects $v_{j}^{i}(u), g_{1}^{i j}(u)$, and $g_{2}^{i j}(u)$ become diagonal. Moreover, in these coordinates, the $i$ th eigenvalue of $v_{j}^{i}(u)$ depends only on the coordinate $R^{i}$. In the case when all the eigenvalues are nonconstant, they can be introduced as new coordinates. In these new coordinates, $\tilde{v}_{j}^{i}(R)=\operatorname{diag}\left(R^{1}, \ldots, R^{N}\right)$ and $\tilde{g}_{2}^{i j}(R)=$ $\operatorname{diag}\left(g^{1}(R), \ldots, g^{N}(R)\right), \tilde{g}_{1}^{i j}(R)=\operatorname{diag}\left(R^{1} g^{1}(R), \ldots, R^{N} g^{N}(R)\right)$. 
In this paper, we unfortunately prove that, in the general case, Theorem 2.5 is not true, and, correspondingly, it is not a criterion of compatibility of flat metrics. Generally speaking, compatibility of flat metrics does not follow from the vanishing of the corresponding Nijenhuis tensor. The corresponding counterexamples will be presented in Section 7 . We also prove that, in the general case, Theorem 2.5 is actually a criterion of almost compatibility of flat metrics that does not guarantee compatibility of the corresponding nondegenerate local Poisson structures of hydrodynamic type. But if the spectrum of $v_{j}^{i}(u)$ is simple, that is, all the eigenvalues are distinct, then we prove that Theorem 2.5 is not only true but also can be essentially generalized to the case of arbitrary compatible Riemannian or pseudo-Riemannian metrics, in particular, the especially important cases in the theory of systems of hydrodynamic type; namely, the cases of metrics of constant Riemannian curvature or the metrics generating the general nonlocal Poisson structures of hydrodynamic type.

Namely, we prove the following theorems for any pseudo-Riemannian metrics (not only for flat metrics as in Theorem 2.5).

THEOREM 2.6. (1) If, for any linear combination (1.6) of two metrics $g_{1}^{i j}(u)$ and $g_{2}^{i j}(u)$, condition (1.7) is fulfilled, then the Nijenhuis tensor of the affinor

$$
v_{j}^{i}(u)=g_{1}^{i s}(u) g_{2, s j}(u)
$$

vanishes. Thus, for any two compatible or almost compatible metrics, the corresponding Nijenhuis tensor always vanishes.

(2) If a pair of metrics $g_{1}^{i j}(u)$ and $g_{2}^{i j}(u)$ is nonsingular, that is, roots of the equation

$$
\operatorname{det}\left(g_{1}^{i j}(u)-\lambda g_{2}^{i j}(u)\right)=0
$$

are distinct, then it follows from the vanishing of the Nijenhuis tensor of the affinor $v_{j}^{i}(u)=g_{1}^{i s}(u) g_{2, s j}(u)$ that the metrics $g_{1}^{i j}(u)$ and $g_{2}^{i j}(u)$ are compatible. Thus, a nonsingular pair of metrics is compatible if and only if the metrics are almost compatible.

THEOREM 2.7. Any nonsingular pair of metrics is compatible if and only if there exist local coordinates $u=\left(u^{1}, \ldots, u^{N}\right)$ such that $g_{2}^{i j}(u)=g^{i}(u) \delta^{i j}$ and $g_{1}^{i j}(u)=f^{i}\left(u^{i}\right) g^{i}(u) \delta^{i j}$, where $f^{i}\left(u^{i}\right), i=1, \ldots, N$, are arbitrary (generally speaking, complex) functions of single variables (of course, the functions $f^{i}\left(u^{i}\right)$ are not identically equal to zero, and, for nonsingular pairs of metrics, all these functions must be distinct; and they cannot be equal to one another if they are 
constants but, nevertheless, in this special case, the metrics will also be compatible).

Sections 3 and 4 are devoted to the proof of Theorems 2.6 and 2.7.

\section{Almost compatible metrics and the Nijenhuis tensor}

Let us consider two arbitrary contravariant Riemannian or pseudoRiemannian metrics $g_{1}^{i j}(u)$ and $g_{2}^{i j}(u)$, and also the corresponding coefficients of the Levi-Civita connections $\Gamma_{1, k}^{i j}(u)$ and $\Gamma_{2, k}^{i j}(u)$.

We introduce the tensor

$$
\begin{aligned}
M^{i j k}(u)= & g_{1}^{i s}(u) \Gamma_{2, s}^{j k}(u)-g_{2}^{j s}(u) \Gamma_{1, s}^{i k}(u) \\
& -g_{1}^{j s}(u) \Gamma_{2, s}^{i k}(u)+g_{2}^{i s}(u) \Gamma_{1, s}^{j k}(u) .
\end{aligned}
$$

It follows from the following representation that $M^{i j k}(u)$ is actually a tensor:

$$
\begin{aligned}
M^{i j k}(u)= & g_{1}^{i s}(u) g_{2}^{j p}(u)\left(\Gamma_{2, p s}^{k}(u)-\Gamma_{1, p s}^{k}(u)\right) \\
& -g_{1}^{j s}(u) g_{2}^{i p}(u)\left(\Gamma_{2, p s}^{k}(u)-\Gamma_{1, p s}^{k}(u)\right) .
\end{aligned}
$$

Lemma 3.1. The tensor $M^{i j k}(u)$ vanishes if and only if the metrics $g_{1}^{i j}(u)$ and $g_{2}^{i j}(u)$ are almost compatible.

Proof. Recall that the functions $\Gamma_{k}^{i j}(u)$ define the Christoffel symbols of the Levi-Civita connection for a contravariant metric $g^{i j}(u)$ if and only if the following relations are fulfilled:

$$
\frac{\partial g^{i j}}{\partial u^{k}}+\Gamma_{k}^{i j}(u)+\Gamma_{k}^{j i}(u)=0
$$

that is, the connection is compatible with the metric; and

$$
g^{i s}(u) \Gamma_{s}^{j k}(u)=g^{j s}(u) \Gamma_{s}^{i k}(u),
$$

that is, the connection is symmetric.

If $g^{i j}(u)$ and $\Gamma_{k}^{i j}(u)$ are defined by formulas (1.6) and (1.7), respectively, then linear relation (3.3) is automatically fulfilled and relation (3.4) is exactly equivalent to the relation $M^{i j k}(u)=0$. 
We introduce the affinor

$$
v_{j}^{i}(u)=g_{1}^{i s}(u) g_{2, s j}(u)
$$

and consider the Nijenhuis tensor of this affinor

$$
N_{i j}^{k}(u)=v_{i}^{s}(u) \frac{\partial v_{j}^{k}}{\partial u^{s}}-v_{j}^{s}(u) \frac{\partial v_{i}^{k}}{\partial u^{s}}+v_{s}^{k}(u) \frac{\partial v_{i}^{s}}{\partial u^{j}}-v_{s}^{k}(u) \frac{\partial v_{j}^{s}}{\partial u^{i}}
$$

following [13], where the affinor $v_{j}^{i}(u)$ and its Nijenhuis tensor were similarly considered for two flat metrics.

THEOREM 3.2. Any two metrics $g_{1}^{i j}(u)$ and $g_{2}^{i j}(u)$ are almost compatible if and only if the corresponding Nijenhuis tensor $N_{i j}^{k}(u)$ (3.6) vanishes.

LEMMA 3.3. The following identities are always fulfilled:

$$
\begin{aligned}
& g_{1, s p}(u) N_{r q}^{p}(u) g_{2}^{r i}(u) g_{2}^{q j}(u) g_{2}^{s k}(u) \\
& =M^{k j i}(u)+M^{i k j}(u)+M^{i j k}(u), \\
& 2\left(M^{i k j}(u)+M^{i j k}(u)\right)=g_{1, s p}(u) N_{r q}^{p}(u) g_{2}^{r i}(u) g_{2}^{q j}(u) g_{2}^{s k}(u) \\
& +g_{1, s p}(u) N_{r q}^{p}(u) g_{2}^{r i}(u) g_{2}^{q k}(u) g_{2}^{s j}(u), \\
& 2 M^{k j i}(u)=g_{1, s p}(u) N_{r q}^{p}(u) g_{2}^{r i}(u) g_{2}^{q j}(u) g_{2}^{s k}(u) \\
& -g_{1, s p}(u) N_{r q}^{p}(u) g_{2}^{r i}(u) g_{2}^{q k}(u) g_{2}^{s j}(u) \text {. }
\end{aligned}
$$

Proof. In the following calculations, using many times both relations (3.3) and (3.4) for both the metrics $g_{1}^{i j}(u)$ and $g_{2}^{i j}(u)$, we have

$$
\begin{aligned}
N_{i j}^{k}(u)= & v_{i}^{s} \frac{\partial v_{j}^{k}}{\partial u^{s}}-v_{j}^{s} \frac{\partial v_{i}^{k}}{\partial u^{s}}+v_{s}^{k} \frac{\partial v_{i}^{s}}{\partial u^{j}}-v_{s}^{k} \frac{\partial v_{j}^{s}}{\partial u^{i}} \\
= & g_{1}^{s p} g_{2, p i} \frac{\partial}{\partial u^{s}}\left(g_{1}^{k l} g_{2, l j}\right)-g_{1}^{s p} g_{2, p j} \frac{\partial}{\partial u^{s}}\left(g_{1}^{k l} g_{2, l i}\right) \\
& +g_{1}^{k p} g_{2, p s} \frac{\partial}{\partial u^{j}}\left(g_{1}^{s l} g_{2, l i}\right)-g_{1}^{k p} g_{2, p s} \frac{\partial}{\partial u^{i}}\left(g_{1}^{s l} g_{2, l j}\right)
\end{aligned}
$$




$$
\begin{aligned}
& =-g_{1}^{s p} g_{2, p i} g_{2, l j}\left(\Gamma_{1, s}^{k l}+\Gamma_{1, s}^{l k}\right)+g_{1}^{s p} g_{2, p i} g_{1}^{k l} g_{2, l r} g_{2, t j}\left(\Gamma_{2, s}^{r t}+\Gamma_{2, s}^{t r}\right) \\
& +g_{1}^{s p} g_{2, p j} g_{2, l i}\left(\Gamma_{1, s}^{k l}+\Gamma_{1, s}^{l k}\right)-g_{1}^{s p} g_{2, p j} g_{1}^{k l} g_{2, l r} g_{2, t i}\left(\Gamma_{2, s}^{r t}+\Gamma_{2, s}^{t r}\right) \\
& -g_{1}^{k p} g_{2, p s} g_{2, l i}\left(\Gamma_{1, j}^{s l}+\Gamma_{1, j}^{l s}\right)+g_{1}^{k p} g_{2, p s} g_{1}^{s l} g_{2, l r} g_{2, t i}\left(\Gamma_{2, j}^{r t}+\Gamma_{2, j}^{t r}\right) \\
& +g_{1}^{k p} g_{2, p s} g_{2, l j}\left(\Gamma_{1, i}^{s l}+\Gamma_{1, i}^{l s}\right)-g_{1}^{k p} g_{2, p s} g_{1}^{s l} g_{2, l r} g_{2, t j}\left(\Gamma_{2, i}^{r t}+\Gamma_{2, i}^{t r}\right), \\
& N_{i j}^{k} g_{2}^{i n} g_{2}^{j m}=-g_{1}^{s n}\left(\Gamma_{1, s}^{k m}+\Gamma_{1, s}^{m k}\right)+g_{1}^{s n} g_{1}^{k l} g_{2, l r}\left(\Gamma_{2, s}^{r m}+\Gamma_{2, s}^{m r}\right) \\
& +g_{1}^{s m}\left(\Gamma_{1, s}^{k n}+\Gamma_{1, s}^{n k}\right)-g_{1}^{s m} g_{1}^{k l} g_{2, l r}\left(\Gamma_{2, s}^{r n}+\Gamma_{2, s}^{n r}\right) \\
& -g_{1}^{k p} g_{2, p s} g_{2}^{j m}\left(\Gamma_{1, j}^{s n}+\Gamma_{1, j}^{n s}\right)+g_{1}^{k p} g_{2, p s} g_{1}^{s l} g_{2, l r} g_{2}^{j m}\left(\Gamma_{2, j}^{r n}+\Gamma_{2, j}^{n r}\right) \\
& +g_{1}^{k p} g_{2, p s} g_{2}^{i n}\left(\Gamma_{1, i}^{s m}+\Gamma_{1, i}^{m s}\right)-g_{1}^{k p} g_{2, p s} g_{1}^{s l} g_{2, l r} g_{2}^{i n}\left(\Gamma_{2, i}^{r m}+\Gamma_{2, i}^{m r}\right) \\
& =-g_{1}^{s n} \Gamma_{1, s}^{k m}+g_{1}^{s n} g_{1}^{k l} g_{2, l r}\left(\Gamma_{2, s}^{r m}+\Gamma_{2, s}^{m r}\right) \\
& +g_{1}^{s m} \Gamma_{1, s}^{k n}-g_{1}^{s m} g_{1}^{k l} g_{2, l r}\left(\Gamma_{2, s}^{r n}+\Gamma_{2, s}^{n r}\right) \\
& -g_{1}^{k p} g_{2, p s} g_{2}^{j m}\left(\Gamma_{1, j}^{s n}+\Gamma_{1, j}^{n s}\right)+g_{1}^{k p} g_{2, p s} g_{1}^{s j} \Gamma_{2, j}^{m n} \\
& +g_{1}^{k p} g_{2, p s} g_{2}^{i n}\left(\Gamma_{1, i}^{s m}+\Gamma_{1, i}^{m s}\right)-g_{1}^{k p} g_{2, p s} g_{1}^{s i} \Gamma_{2, i}^{n m}, \\
& g_{1, q k} N_{i j}^{k} g_{2}^{i n} g_{2}^{j m}=-\Gamma_{1, q}^{n m}+g_{1}^{s n} g_{2, q r}\left(\Gamma_{2, s}^{r m}+\Gamma_{2, s}^{m r}\right) \\
& +\Gamma_{1, q}^{m n}-g_{1}^{s m} g_{2, q r}\left(\Gamma_{2, s}^{r n}+\Gamma_{2, s}^{n r}\right)-g_{2, q s} g_{2}^{j m}\left(\Gamma_{1, j}^{s n}+\Gamma_{1, j}^{n s}\right) \\
& +g_{2, q s} g_{1}^{s j} \Gamma_{2, j}^{m n}+g_{2, q s} g_{2}^{i n}\left(\Gamma_{1, i}^{s m}+\Gamma_{1, i}^{m s}\right)-g_{2, q s} g_{1}^{s i} \Gamma_{2, i}^{n m},
\end{aligned}
$$

and, finally,

$$
\begin{aligned}
g_{1, q k} N_{i j}^{k} g_{2}^{i n} g_{2}^{j m} g_{2}^{t q}= & -g_{2}^{t q} \Gamma_{1, q}^{n m}+g_{1}^{s n}\left(\Gamma_{2, s}^{t m}+\Gamma_{2, s}^{m t}\right) \\
& +g_{2}^{t q} \Gamma_{1, q}^{m n}-g_{1}^{s m}\left(\Gamma_{2, s}^{t n}+\Gamma_{2, s}^{n t}\right)-g_{2}^{j m}\left(\Gamma_{1, j}^{t n}+\Gamma_{1, j}^{n t}\right) \\
& +g_{1}^{t j} \Gamma_{2, j}^{m n}+g_{2}^{i n}\left(\Gamma_{1, i}^{t m}+\Gamma_{1, i}^{m t}\right)-g_{1}^{t i} \Gamma_{2, i}^{n m} \\
= & M^{t m n}+M^{n t m}+M^{n m t} .
\end{aligned}
$$

Note that the tensor $M^{i j k}(u),(3.1)$, is skew-symmetric with respect to the indices $i$ and $j$. Permuting the indices $k$ and $j$ in formula (3.7) and adding the corresponding relation to (3.7), we obtain (3.8). Formula (3.9) follows from (3.7) and (3.8) straightforward. 
Corollary 3.4. The tensor $M^{i j k}(u)$ vanishes if and only if the Nijenhuis tensor (3.6) vanishes.

In $[25,26,27,28,29]$, the author studied special reductions in the general problem on compatible flat metrics, namely, the reductions connected with the associativity equations, that is, the following general ansatz in formula (2.24):

$$
h^{i}(v)=\eta^{i s} \frac{\partial \Phi}{\partial v^{s}}
$$

where $\Phi\left(v^{1}, \ldots, v^{N}\right)$ is a function of $N$ variables.

Correspondingly, in this case, the metrics have the form

$$
g_{1}^{i j}(v)=\eta^{i j}, \quad g_{2}^{i j}(v)=\eta^{i s} \eta^{j p} \frac{\partial^{2} \Phi}{\partial v^{s} \partial v^{p}}
$$

THEOREM $3.5[26,28,29]$. If metrics (3.13) are almost compatible, then they are compatible. Moreover, in this case, the metric $g_{2}^{i j}(v)$ also is necessarily flat, that is, metrics (3.13) form a flat pencil of metrics. The condition of almost compatibility for metrics (3.13) has the form

$$
\eta^{s p} \frac{\partial^{2} \Phi}{\partial v^{p} \partial v^{i}} \frac{\partial^{3} \Phi}{\partial v^{s} \partial v^{j} \partial v^{k}}=\eta^{s p} \frac{\partial^{2} \Phi}{\partial v^{p} \partial v^{k}} \frac{\partial^{3} \Phi}{\partial v^{s} \partial v^{j} \partial v^{i}}
$$

and coincides with the condition of compatible deformation of two Frobenius algebras (this condition was derived and studied by the author in [26, 27, 28, 29]).

In particular, in $[26,27,28,29]$, it is proved that, in the two-component case $(N=2)$, for $\eta^{i j}=\varepsilon^{i} \delta^{i j}, \varepsilon^{i}= \pm 1$, condition (3.14) is equivalent to the following linear second-order partial differential equation with constant coefficients:

$$
\alpha\left(\varepsilon^{1} \frac{\partial^{2} \Phi}{\partial\left(v^{1}\right)^{2}}-\varepsilon^{2} \frac{\partial^{2} \Phi}{\partial\left(v^{2}\right)^{2}}\right)=\beta \frac{\partial^{2} \Phi}{\partial v^{1} \partial v^{2}}
$$

where $\alpha$ and $\beta$ are arbitrary constants that are not equal to zero simultaneously.

\section{Compatible metrics and the Nijenhuis tensor}

We prove the second part of Theorem 2.6. In Section 3, we particularly proved that it always follows from compatibility (moreover, even from 
almost compatibility) of metrics that the corresponding Nijenhuis tensor vanishes (Theorem 3.2).

Assume that a pair of metrics $g_{1}^{i j}(u)$ and $g_{2}^{i j}(u)$ is nonsingular, that is, the eigenvalues of this pair of metrics are distinct. Furthermore, assume that the corresponding Nijenhuis tensor vanishes. We prove that, in this case, the metrics $g_{1}^{i j}(u)$ and $g_{2}^{i j}(u)$ are compatible (their almost compatibility follows from Theorem 3.2).

It is obvious that eigenvalues of the pair of metrics $g_{1}^{i j}(u)$ and $g_{2}^{i j}(u)$ coincide with eigenvalues of the affinor $v_{j}^{i}(u)$. But it is well known that if all eigenvalues of an affinor are distinct, then it always follows from the vanishing of the Nijenhuis tensor of this affinor that there exist local coordinates such that, in these coordinates, the affinor reduces to a diagonal form in the corresponding neighbourhood [32] (see also [20]).

So, further, we can consider that the affinor $v_{j}^{i}(u)$ is diagonal in the local coordinates $u^{1}, \ldots, u^{N}$, that is,

$$
v_{j}^{i}(u)=\lambda^{i}(u) \delta_{j}^{i}
$$

where there is no summation over the index $i$. By assumption, the eigenvalues $\lambda^{i}(u), i=1, \ldots, N$, coinciding with eigenvalues of the pair of metrics $g_{1}^{i j}(u)$ and $g_{2}^{i j}(u)$, are distinct

$$
\lambda^{i} \neq \lambda^{j} \quad \text { if } i \neq j
$$

LEMMA 4.1. If the affinor $v_{j}^{i}(u)$ in (3.5) is diagonal in certain local coordinates and all its eigenvalues are distinct, then, in these coordinates, the metrics $g_{1}^{i j}(u)$ and $g_{2}^{i j}(u)$ are also necessarily diagonal.

Proof. Actually, we have

$$
g_{1}^{i j}(u)=\lambda^{i}(u) g_{2}^{i j}(u)
$$

It follows from the symmetry of the metrics $g_{1}^{i j}(u)$ and $g_{2}^{i j}(u)$ that, for any indices $i$ and $j$,

$$
\left(\lambda^{i}(u)-\lambda^{j}(u)\right) g_{2}^{i j}(u)=0
$$

where tehre is no summation over indices, that is,

$$
g_{2}^{i j}(u)=g_{1}^{i j}(u)=0 \quad \text { if } i \neq j .
$$


LEMMA 4.2. Let an affinor $w_{j}^{i}(u)$ be diagonal in certain local coordinates $u=$ $\left(u^{1}, \ldots, u^{N}\right)$, that is, $w_{j}^{i}(u)=\mu^{i}(u) \delta_{j}^{i}$.

(1) If all the eigenvalues $\mu^{i}(u), i=1, \ldots, N$, of the diagonal affinor are distinct, that is, $\mu^{i}(u) \neq \mu^{j}(u)$ for $i \neq j$, then the Nijenhuis tensor of this affinor vanishes if and only if the ith eigenvalue $\mu^{i}(u)$ depends only on the coordinate $u^{i}$.

(2) If all the eigenvalues coincide, then the Nijenhuis tensor vanishes.

(3) In the general case of an arbitrary diagonal affinor $w_{j}^{i}(u)=\mu^{i}(u) \delta_{j}^{i}$, the Nijenhuis tensor vanishes if and only if

$$
\frac{\partial \mu^{i}}{\partial u^{j}}=0
$$

for all indices $i$ and $j$ such that $\mu^{i}(u) \neq \mu^{j}(u)$.

Proof. Actually, for any diagonal affinor $w_{j}^{i}(u)=\mu^{i}(u) \delta_{j}^{i}$, the Nijenhuis tensor $N_{i j}^{k}(u)$ has the form

$$
N_{i j}^{k}(u)=\left(\mu^{i}-\mu^{k}\right) \frac{\partial \mu^{j}}{\partial u^{i}} \delta^{k j}-\left(\mu^{j}-\mu^{k}\right) \frac{\partial \mu^{i}}{\partial u^{j}} \delta^{k i}
$$

(no summation over indices). Thus, the Nijenhuis tensor vanishes if and only if, for any indices $i$ and $j$,

$$
\left(\mu^{i}(u)-\mu^{j}(u)\right) \frac{\partial \mu^{i}}{\partial u^{j}}=0,
$$

where there is no summation over indices.

It follows from Lemmas 4.1 and 4.2 that, for any nonsingular pair of almost compatible metrics, there always exist local coordinates in which the metrics have the form

$$
g_{2}^{i j}(u)=g^{i}(u) \delta^{i j}, \quad g_{1}^{i j}(u)=\lambda^{i}\left(u^{i}\right) g^{i}(u) \delta^{i j}, \quad \lambda^{i}=\lambda^{i}\left(u^{i}\right), i=1, \ldots, N .
$$

Moreover, we immediately derive that any pair of diagonal metrics of the form $g_{2}^{i j}(u)=g^{i}(u) \delta^{i j}$ and $g_{1}^{i j}(u)=f^{i}\left(u^{i}\right) g^{i}(u) \delta^{i j}$ for any nonzero functions $f^{i}\left(u^{i}\right), i=1, \ldots, N$, (here they can be, e.g., coinciding nonzero constants, i.e., the pair of metrics may be singular) is almost compatible since the corresponding Nijenhuis tensor always vanishes for any pair of metrics of this form.

We prove now that any pair of metrics of this form is always compatible. Then, Theorems 2.6 and 2.7 will be completely proved. 
Consider two diagonal metrics of the form $g_{2}^{i j}(u)=g^{i}(u) \delta^{i j}$ and $g_{1}^{i j}(u)$ $=f^{i}\left(u^{i}\right) g^{i}(u) \delta^{i j}$, where $f^{i}\left(u^{i}\right), i=1, \ldots, N$, are arbitrary (possibly, complex) nonzero functions of single variables, and consider their arbitrary linear combination

$$
g^{i j}(u)=\left(\lambda_{2}+\lambda_{1} f^{i}\left(u^{i}\right)\right) g^{i}(u) \delta^{i j},
$$

where $\lambda_{1}$ and $\lambda_{2}$ are arbitrary constants such that $\operatorname{det}\left(g^{i j}(u)\right) \not \equiv 0$.

We prove that relation (1.8) is always fulfilled for the corresponding tensors of Riemannian curvature.

Recall that, for any diagonal metric, $\Gamma_{j k}^{i}(u)=0$ if all the indices $i, j$, $k$ are distinct. Correspondingly, $R_{k l}^{i j}(u)=0$ if all the indices $i, j, k, l$ are distinct. Besides, as a result of the well-known symmetries of the tensor of Riemannian curvature, we have

$$
\begin{gathered}
R_{k l}^{i i}(u)=R_{k k}^{i j}(u)=0, \\
R_{i l}^{i j}(u)=-R_{l i}^{i j}(u)=R_{l i}^{j i}(u)=-R_{i l}^{j i}(u) .
\end{gathered}
$$

Thus, it is sufficient to prove relation (1.8) only for the following components of the corresponding tensors of Riemannian curvature: $R_{i l}^{i j}(u)$, where $i \neq j, i \neq l$.

For an arbitrary diagonal metric $g_{2}^{i j}(u)=g^{i}(u) \delta^{i j}$, we have

$$
\begin{aligned}
\Gamma_{2, i k}^{i}(u) & =\Gamma_{2, k i}^{i}(u)=-\frac{1}{2 g^{i}(u)} \frac{\partial g^{i}}{\partial u^{k}} \text { for any } i, k, \\
\Gamma_{2, j j}^{i}(u) & =\frac{1}{2} \frac{g^{i}(u)}{\left(g^{j}(u)\right)^{2}} \frac{\partial g^{j}}{\partial u^{i}}, \quad i \neq j, \\
R_{2, i l}^{i j}(u) & =g^{i}(u) R_{2, i l}^{j}(u) \\
& =g^{i}(u)\left(\frac{\partial \Gamma_{2, i l}^{j}}{\partial u^{i}}-\frac{\partial \Gamma_{2, i i}^{j}}{\partial u^{l}}+\sum_{s=1}^{N} \Gamma_{2, s i}^{j}(u) \Gamma_{2, i l}^{s}(u)-\sum_{s=1}^{N} \Gamma_{2, s l}^{j}(u) \Gamma_{2, i i}^{s}(u)\right) .
\end{aligned}
$$

It is necessary to consider the following two different cases separately. 
Case $1(j \neq l)$

The corresponding components of the Riemannian curvature tensor have the form

$$
\begin{aligned}
R_{2, i l}^{i j}(u)= & g^{i}(u)\left(-\frac{\partial \Gamma_{2, i i}^{j}}{\partial u^{l}}+\Gamma_{2, i i}^{j}(u) \Gamma_{2, i l}^{i}(u)\right. \\
& \left.-\Gamma_{2, j l}^{j}(u) \Gamma_{2, i i}^{j}(u)-\Gamma_{2, l l}^{j}(u) \Gamma_{2, i i}^{l}(u)\right) \\
= & -\frac{1}{2} g^{i}(u) \frac{\partial}{\partial u^{l}}\left(\frac{g^{j}(u)}{\left(g^{i}(u)\right)^{2}} \frac{\partial g^{i}}{\partial u^{j}}\right)-\frac{1}{4} \frac{g^{j}(u)}{\left(g^{i}(u)\right)^{2}} \frac{\partial g^{i}}{\partial u^{j}} \frac{\partial g^{i}}{\partial u^{l}} \\
& +\frac{1}{4 g^{i}(u)} \frac{\partial g^{i}}{\partial u^{j}} \frac{\partial g^{j}}{\partial u^{l}}-\frac{1}{4} \frac{g^{j}(u)}{g^{i}(u) g^{l}(u)} \frac{\partial g^{l}}{\partial u^{j}} \frac{\partial g^{i}}{\partial u^{l}} .
\end{aligned}
$$

Respectively, for the metric

$$
g^{i j}(u)=\left(\lambda_{2}+\lambda_{1} f^{i}\left(u^{i}\right)\right) g^{i}(u) \delta^{i j},
$$

we obtain (here, we use that all the indices $i, j, l$ are distinct) the following relation:

$$
\begin{aligned}
& R_{i l}^{i j}(u)=\left(\lambda_{2}+\lambda_{1} f^{j}\left(u^{j}\right)\right)[-\frac{1}{2} g^{i}(u) \frac{\partial}{\partial u^{l}}\left(\frac{g^{j}(u)}{\left(g^{i}(u)\right)^{2}} \frac{\partial g^{i}}{\partial u^{j}}\right) \\
&-\frac{1}{4} \frac{g^{j}(u)}{\left(g^{i}(u)\right)^{2}} \frac{\partial g^{i}}{\partial u^{j}} \frac{\partial g^{i}}{\partial u^{l}}+\frac{1}{4 g^{i}(u)} \frac{\partial g^{i}}{\partial u^{j}} \frac{\partial g^{j}}{\partial u^{l}} \\
&\left.-\frac{1}{4} \frac{g^{j}(u)}{g^{i}(u) g^{l}(u)} \frac{\partial g^{l}}{\partial u^{j}} \frac{\partial g^{i}}{\partial u^{l}}\right] \\
&=\lambda_{1} R_{1, i l}^{i j}(u)+\lambda_{2} R_{2, i l}^{i j}(u) .
\end{aligned}
$$

Case $2(j=l)$

The corresponding components of the Riemannian curvature tensor have the form

$$
\begin{aligned}
R_{2, i j}^{i j}(u)=g^{i}(u) & \left(\frac{\partial \Gamma_{2, i j}^{j}}{\partial u^{i}}-\frac{\partial \Gamma_{2, i i}^{j}}{\partial u^{j}}+\Gamma_{2, i i}^{j}(u) \Gamma_{2, i j}^{i}(u)\right. \\
& \left.+\Gamma_{2, j i}^{j}(u) \Gamma_{2, i j}^{j}(u)-\sum_{s=1}^{N} \Gamma_{2, s j}^{j}(u) \Gamma_{2, i i}^{s}(u)\right)
\end{aligned}
$$


Compatible flat metrics

$$
\begin{aligned}
= & -\frac{1}{2} g^{i}(u) \frac{\partial}{\partial u^{i}}\left(\frac{1}{g^{j}(u)} \frac{\partial g^{j}}{\partial u^{i}}\right)-\frac{1}{2} g^{i}(u) \frac{\partial}{\partial u^{j}}\left(\frac{g^{j}(u)}{\left(g^{i}(u)\right)^{2}} \frac{\partial g^{i}}{\partial u^{j}}\right) \\
& -\frac{1}{4} \frac{g^{j}(u)}{\left(g^{i}(u)\right)^{2}} \frac{\partial g^{i}}{\partial u^{j}} \frac{\partial g^{i}}{\partial u^{j}}+\frac{1}{4} \frac{g^{i}(u)}{\left(g^{j}(u)\right)^{2}} \frac{\partial g^{j}}{\partial u^{i}} \frac{\partial g^{j}}{\partial u^{i}} \\
& -\frac{1}{4 g^{j}(u)} \frac{\partial g^{j}}{\partial u^{i}} \frac{\partial g^{i}}{\partial u^{i}}+\sum_{s \neq i} \frac{1}{4} \frac{g^{s}(u)}{g^{i}(u) g^{j}(u)} \frac{\partial g^{j}}{\partial u^{s}} \frac{\partial g^{i}}{\partial u^{s}} .
\end{aligned}
$$

Respectively, for the metric

$$
g^{i j}(u)=\left(\lambda_{2}+\lambda_{1} f^{i}\left(u^{i}\right)\right) g^{i}(u) \delta^{i j}
$$

we obtain (here, we use that the indices $i$ and $j$ are distinct) the following relation:

$$
\begin{aligned}
R_{i j}^{i j}(u)= & -\frac{1}{2}\left(\lambda_{2}+\lambda_{1} f^{i}\left(u^{i}\right)\right) g^{i}(u) \frac{\partial}{\partial u^{i}}\left(\frac{1}{g^{j}(u)} \frac{\partial g^{j}}{\partial u^{i}}\right) \\
& -\frac{1}{2} g^{i}(u) \frac{\partial}{\partial u^{j}}\left(\frac{\left(\lambda_{2}+\lambda_{1} f^{j}\left(u^{j}\right)\right) g^{j}(u)}{\left(g^{i}(u)\right)^{2}} \frac{\partial g^{i}}{\partial u^{j}}\right) \\
& -\frac{1}{4}\left(\lambda_{2}+\lambda_{1} f^{j}\left(u^{j}\right)\right) \frac{g^{j}(u)}{\left(g^{i}(u)\right)^{2}} \frac{\partial g^{i}}{\partial u^{j}} \frac{\partial g^{i}}{\partial u^{j}} \\
& +\frac{1}{4}\left(\lambda_{2}+\lambda_{1} f^{i}\left(u^{i}\right)\right) \frac{g^{i}(u)}{\left(g^{j}(u)\right)^{2}} \frac{\partial g^{j}}{\partial u^{i}} \frac{\partial g^{j}}{\partial u^{i}} \\
& -\frac{1}{4 g^{j}(u)} \frac{\partial g^{j}}{\partial u^{i}} \frac{\partial\left(\left(\lambda_{2}+\lambda_{1} f^{i}\left(u^{i}\right)\right) g^{i}\right)}{\partial u^{i}} \\
& +\frac{1}{4 g^{i}(u)} \frac{\partial g^{i}}{\partial u^{j}} \frac{\partial\left(\left(\lambda_{2}+\lambda_{1} f^{j}\left(u^{j}\right)\right) g^{j}\right)}{\partial u^{j}} \\
& +\sum_{s \neq i, s \neq j} \frac{1}{4} \frac{\left(\lambda_{2}+\lambda_{1} f^{s}\left(u^{s}\right)\right) g^{s}(u)}{g^{i}(u) g^{j}(u)} \frac{\partial g^{j}}{\partial u^{s}} \frac{\partial g^{i}}{\partial u^{s}} \\
= & \lambda_{1} R_{1, i j}^{i j}(u)+\lambda_{2} R_{2, i j}^{i j}(u) .
\end{aligned}
$$


Theorems 2.6 and 2.7 are proved. Thus, the complete explicit description of nonsingular pairs of compatible and almost compatible metrics is obtained.

\section{Equations for nonsingular pairs of compatible flat metrics}

Now, consider in detail the problem of nonsingular pairs of compatible flat metrics. It follows from Theorem 2.7 that it is sufficient to classify all pairs of flat metrics of the following special diagonal form $g_{2}^{i j}(u)=$ $g^{i}(u) \delta^{i j}$ and $g_{1}^{i j}(u)=f^{i}\left(u^{i}\right) g^{i}(u) \delta^{i j}$, where $f^{i}\left(u^{i}\right), i=1, \ldots, N$, are arbitrary (possibly, complex) functions of single variables.

The problem of description of diagonal flat metrics, that is, flat metrics $g_{2}^{i j}(u)=g^{i}(u) \delta^{i j}$, is a classical problem of differential geometry. This problem is equivalent to the problem of description of curvilinear orthogonal coordinate systems in an $\mathrm{N}$-dimensional pseudo-Euclidean space, and it was studied in detail and was mainly solved at the beginning of the 20th century (see [4]). Locally, such coordinate systems are determined by $N(N-1) / 2$ arbitrary functions of two variables. Recently, Zakharov showed that the Lamé equations describing curvilinear orthogonal coordinate systems can be integrated by the inverse scattering method [36] (see also an algebraic-geometric approach in [21]).

The condition that the metric $g_{1}^{i j}(u)=f^{i}\left(u^{i}\right) g^{i}(u) \delta^{i j}$ is also flat exactly gives $N(N-1) / 2$ additional equations linear with respect to the functions $f^{i}\left(u^{i}\right)$. Note that, in this case, components (4.13) of the corresponding tensor of Riemannian curvature automatically vanish as a result of formula (4.15). And the vanishing of components (4.16) gives the corresponding $N(N-1) / 2$ equations. In particular, in the case $N=2$, this completely solves the problem of description of nonsingular pairs of compatible two-component flat metrics. In the next section, we present this complete description. It is also very interesting to classify all the $\mathrm{N}$ orthogonal curvilinear coordinate systems in a pseudo-Euclidean space (or, in other words, to classify the corresponding functions $g^{i}(u)$ ) such that the functions $f^{i}\left(u^{i}\right)=\left(u^{i}\right)^{n}$ define the corresponding compatible flat metrics (for $n=1, n=1,2, n=1,2,3$, and so on, respectively).

THEOREM 5.1. Any nonsingular pair of compatible flat metrics is described by the following integrable nonlinear system, which is the special reduction of the following Lamé equations:

$$
\begin{gathered}
\frac{\partial \beta_{i j}}{\partial u^{k}}=\beta_{i k} \beta_{k j}, \quad i \neq j, i \neq k, j \neq k, \\
\frac{\partial \beta_{i j}}{\partial u^{i}}+\frac{\partial \beta_{j i}}{\partial u^{j}}+\sum_{s \neq i, s \neq j} \beta_{s i} \beta_{s j}=0, \quad i \neq j,
\end{gathered}
$$




$$
\begin{aligned}
\sqrt{f^{i}\left(u^{i}\right) \frac{\partial\left(\sqrt{f^{i}\left(u^{i}\right)} \beta_{i j}\right)}{\partial u^{i}}}+\sqrt{f^{j}\left(u^{j}\right) \frac{\partial\left(\sqrt{f^{j}\left(u^{j}\right)} \beta_{j i}\right)}{\partial u^{j}}} \\
+\sum_{s \neq i, s \neq j} f^{s}\left(u^{s}\right) \beta_{s i} \beta_{s j}=0, \quad i \neq j,
\end{aligned}
$$

where $f^{i}\left(u^{i}\right), i=1, \ldots, N$, are given arbitrary (possibly, complex) functions of single variables (these functions are eigenvalues of the pair of metrics).

Remark 5.2. Equations (5.1) and (5.2) are the famous Lamé equations. Equations (5.3) define a nontrivial nonlinear differential reduction of the Lamé equations.

Proof. Consider the conditions of flatness for the diagonal metrics $g_{2}^{i j}(u)$ $=g^{i}(u) \delta^{i j}$ and $g_{1}^{i j}(u)=f^{i}\left(u^{i}\right) g^{i}(u) \delta^{i j}$, where $f^{i}\left(u^{i}\right), i=1, \ldots, N$, are arbitrary (possibly, complex) functions of the given single variables (but these functions are not identically equal to zero).

As shown in Section 4, for any diagonal metric, it is sufficient to consider the condition $R_{k l}^{i j}(u)=0$ (the condition of flatness for a metric) only for the following components of the tensor of Riemannian curvature: $R_{i l}^{i j}(u)$, where $i \neq j, i \neq l$.

Again as above, for an arbitrary diagonal metric $g_{2}^{i j}(u)=g^{i}(u) \delta^{i j}$, it is necessary to consider the following two different cases separately.

\section{Case $1(j \neq l)$}

In this case the condition of flatness for the metric gives

$$
\begin{aligned}
R_{2, i l}^{i j}(u)= & -\frac{1}{2} g^{i}(u) \frac{\partial}{\partial u^{l}}\left(\frac{g^{j}(u)}{\left(g^{i}(u)\right)^{2}} \frac{\partial g^{i}}{\partial u^{j}}\right)-\frac{1}{4} \frac{g^{j}(u)}{\left(g^{i}(u)\right)^{2}} \frac{\partial g^{i}}{\partial u^{j}} \frac{\partial g^{i}}{\partial u^{l}} \\
& +\frac{1}{4 g^{i}(u)} \frac{\partial g^{i}}{\partial u^{j}} \frac{\partial g^{j}}{\partial u^{l}}-\frac{1}{4} \frac{g^{j}(u)}{g^{i}(u) g^{l}(u)} \frac{\partial g^{l}}{\partial u^{j}} \frac{\partial g^{i}}{\partial u^{l}} \\
= & 0 .
\end{aligned}
$$

Introducing the standard classical notation

$$
\begin{aligned}
& g^{i}(u)=\frac{1}{\left(H_{i}(u)\right)^{2}}, \quad d s^{2}=\sum_{i=1}^{N}\left(H_{i}(u)\right)^{2}\left(d u^{i}\right)^{2}, \\
& \beta_{i k}(u)=\frac{1}{H_{i}(u)} \frac{\partial H_{k}}{\partial u^{i}}, \quad i \neq k,
\end{aligned}
$$


where $H_{i}(u)$ are the Lamé coefficients and $\beta_{i k}(u)$ are the rotation coefficients, we derive that (5.4) are equivalent to the equations

$$
\frac{\partial^{2} H_{i}}{\partial u^{j} \partial u^{k}}=\frac{1}{H_{j}(u)} \frac{\partial H_{i}}{\partial u^{j}} \frac{\partial H_{j}}{\partial u^{k}}+\frac{1}{H_{k}(u)} \frac{\partial H_{k}}{\partial u^{j}} \frac{\partial H_{i}}{\partial u^{k}}
$$

where $i \neq j, i \neq k, j \neq k$. Equations (5.6) are equivalent to equations (5.1).

Case $2(j=l)$

In this case the condition of flatness for the metric gives

$$
\begin{aligned}
R_{2, i j}^{i j}(u)= & -\frac{1}{2} g^{i}(u) \frac{\partial}{\partial u^{i}}\left(\frac{1}{g^{j}(u)} \frac{\partial g^{j}}{\partial u^{i}}\right)-\frac{1}{2} g^{i}(u) \frac{\partial}{\partial u^{j}}\left(\frac{g^{j}(u)}{\left(g^{i}(u)\right)^{2}} \frac{\partial g^{i}}{\partial u^{j}}\right) \\
& -\frac{1}{4} \frac{g^{j}(u)}{\left(g^{i}(u)\right)^{2}} \frac{\partial g^{i}}{\partial u^{j}} \frac{\partial g^{i}}{\partial u^{j}}+\frac{1}{4} \frac{g^{i}(u)}{\left(g^{j}(u)\right)^{2}} \frac{\partial g^{j}}{\partial u^{i}} \frac{\partial g^{j}}{\partial u^{i}} \\
& -\frac{1}{4 g^{j}(u)} \frac{\partial g^{j}}{\partial u^{i}} \frac{\partial g^{i}}{\partial u^{i}}+\sum_{s \neq i} \frac{1}{4} \frac{g^{s}(u)}{g^{i}(u) g^{j}(u)} \frac{\partial g^{j}}{\partial u^{s}} \frac{\partial g^{i}}{\partial u^{s}} \\
= & 0 .
\end{aligned}
$$

Equations (5.7) are equivalent to the following equations:

$$
\begin{aligned}
& \frac{\partial}{\partial u^{i}}\left(\frac{1}{H_{i}(u)} \frac{\partial H_{j}}{\partial u^{i}}\right)+\frac{\partial}{\partial u^{j}}\left(\frac{1}{H_{j}(u)} \frac{\partial H_{i}}{\partial u^{j}}\right) \\
&+\sum_{s \neq i, s \neq j} \frac{1}{\left(H_{S}(u)\right)^{2}} \frac{\partial H_{i}}{\partial u^{s}} \frac{\partial H_{j}}{\partial u^{s}}=0, \quad i \neq j .
\end{aligned}
$$

Equations (5.8) are equivalent to equations (5.2).

The condition that the metric $g_{1}^{i j}(u)=f^{i}\left(u^{i}\right) g^{i}(u) \delta^{i j}$ is also flat gives exactly $N(N-1) / 2$ additional equations (5.3), which are linear with respect to the given functions $f^{i}\left(u^{i}\right)$. Note that, in this case, components (5.4) of the corresponding tensor of Riemannian curvature automatically vanish. And the vanishing of components (5.7) gives the corresponding $N(N-1) / 2$ additional equations. 
Actually, for the metric $g_{1}^{i j}(u)=f^{i}\left(u^{i}\right) g^{i}(u) \delta^{i j}$, we have

$$
\begin{aligned}
\widetilde{H}_{i}(u) & =\frac{H_{i}(u)}{\sqrt{f^{i}\left(u^{i}\right)}}, \\
\tilde{\beta}_{i k}(u) & =\frac{1}{\widetilde{H}_{i}(u)} \frac{\partial \widetilde{H}_{k}}{\partial u^{i}}=\frac{\sqrt{f^{i}\left(u^{i}\right)}}{\sqrt{f^{k}\left(u^{k}\right)}}\left(\frac{1}{H_{i}(u)} \frac{\partial H_{k}}{\partial u^{i}}\right) \\
& =\frac{\sqrt{f^{i}\left(u^{i}\right)}}{\sqrt{f^{k}\left(u^{k}\right)}} \beta_{i k}(u), \quad i \neq k .
\end{aligned}
$$

Respectively, (5.1) are also fulfilled for the rotation coefficients $\tilde{\beta}_{i k}(u)$ and (5.2) for the rotation coefficient $\tilde{\beta}_{i k}(u)$ give (5.3), which can be rewritten as follows (as linear equations with respect to the functions $f^{i}\left(u^{i}\right)$ ):

$$
\begin{aligned}
f^{i}\left(u^{i}\right) & \frac{\partial \beta_{i j}}{\partial u^{i}}+\frac{1}{2}\left(f^{i}\left(u^{i}\right)\right)^{\prime} \beta_{i j}+f^{j}\left(u^{j}\right) \frac{\partial \beta_{j i}}{\partial u^{j}} \\
& +\frac{1}{2}\left(f^{j}\left(u^{j}\right)\right)^{\prime} \beta_{j i}+\sum_{s \neq i, s \neq j} f^{s}\left(u^{s}\right) \beta_{s i} \beta_{s j}=0, \quad i \neq j .
\end{aligned}
$$

\section{Two-component compatible flat metrics}

Here, we present the complete description of nonsingular pairs of twocomponent compatible flat metrics (see also [25, 28, 29], where an integrable four-component nondiagonalizable homogeneous system of hydrodynamic type, describing all the two-component compatible flat metrics, was derived and investigated).

It is shown above (Theorem 2.7 for $N=2$ ) that, for any nonsingular pair of two-component compatible metrics $g_{1}^{i j}(u)$ and $g_{2}^{i j}(u)$, there always exist local coordinates $u^{1}, u^{2}$ such that

$$
\begin{aligned}
& \left(g_{2}^{i j}(u)\right)=\left(\begin{array}{cc}
\frac{\varepsilon^{1}}{\left(b^{1}(u)\right)^{2}} & 0 \\
0 & \frac{\varepsilon^{2}}{\left(b^{2}(u)\right)^{2}}
\end{array}\right), \\
& \left(g_{1}^{i j}(u)\right)=\left(\begin{array}{cc}
\frac{\varepsilon^{1} f^{1}\left(u^{1}\right)}{\left(b^{1}(u)\right)^{2}} & 0 \\
0 & \frac{\varepsilon^{2} f^{2}\left(u^{2}\right)}{\left(b^{2}(u)\right)^{2}}
\end{array}\right),
\end{aligned}
$$


where $\varepsilon^{i}= \pm 1, i=1,2 ; b^{i}(u)$ and $f^{i}\left(u^{i}\right), i=1,2$, are arbitrary nonzero functions of the corresponding variables.

LeMma 6.1. An arbitrary diagonal metric $g_{2}^{i j}(u),(6.1)$, is flat if and only if the functions $b^{i}(u), i=1,2$, are solutions of the following linear system:

$$
\frac{\partial b^{2}}{\partial u^{1}}=\varepsilon^{1} \frac{\partial F}{\partial u^{2}} b^{1}(u), \quad \frac{\partial b^{1}}{\partial u^{2}}=-\varepsilon^{2} \frac{\partial F}{\partial u^{1}} b^{2}(u)
$$

where $F(u)$ is an arbitrary function.

THEOREM 6.2. The metrics $g_{1}^{i j}(u)$ and $g_{2}^{i j}(u),(6.1)$, form a flat pencil of metrics if and only if the functions $b^{i}(u), i=1,2$, are solutions of the linear system (6.2), where the function $F(u)$ is a solution of the following linear equation:

$$
2 \frac{\partial^{2} F}{\partial u^{1} \partial u^{2}}\left(f^{1}\left(u^{1}\right)-f^{2}\left(u^{2}\right)\right)+\frac{\partial F}{\partial u^{2}} \frac{d f^{1}\left(u^{1}\right)}{d u^{1}}-\frac{\partial F}{\partial u^{1}} \frac{d f^{2}\left(u^{2}\right)}{d u^{2}}=0
$$

If eigenvalues of the pair of metrics $g_{1}^{i j}(u)$ and $g_{2}^{i j}(u)$ are distinct and constant, then we can always choose local coordinates such that $f^{1}\left(u^{1}\right)=$ $u^{1}, f^{2}\left(u^{2}\right)=u^{2}$ (see also the remark in [13]). In this case, (6.3) has the form

$$
2 \frac{\partial^{2} F}{\partial u^{1} \partial u^{2}}\left(u^{1}-u^{2}\right)+\frac{\partial F}{\partial u^{2}}-\frac{\partial F}{\partial u^{1}}=0
$$

We continue this recurrent procedure for the metrics $G_{n+1}^{i j}(u)=$ $v_{s}^{i}(u) G_{n}^{s j}(u)$ with the help of the affinor $v_{j}^{i}(u)=u^{i} \delta_{j}^{i}$.

THEOREM 6.3. Three metrics

$$
\left(G_{n}^{i j}(u)\right)=\left(\begin{array}{cc}
\frac{\varepsilon^{1}\left(u^{1}\right)^{n}}{\left(b^{1}(u)\right)^{2}} & 0 \\
0 & \frac{\varepsilon^{2}\left(u^{2}\right)^{n}}{\left(b^{2}(u)\right)^{2}}
\end{array}\right), \quad n=0,1,2
$$

form a flat pencil of metrics (pairwise compatible) if and only if the functions $b^{i}(u), i=1,2$, are solutions of linear system (6.2), where

$$
F(u)=c \ln \left(u^{1}-u^{2}\right)
$$


and $c$ is an arbitrary constant. The metrics $G_{n}^{i j}(u), n=0,1,2,3$, are flat only in the most trivial case when $c=0$ and, respectively, $b^{1}=b^{1}\left(u^{1}\right)$ and $b^{2}=b^{2}\left(u^{2}\right)$.

The metrics $G_{n}^{i j}(u), n=0,1,2$, are flat and the metric $G_{3}^{i j}(u)$ is a metric of nonzero constant Riemannian curvature $K \neq 0$ (in this case, the metrics $G_{n}^{i j}$, $n=0,1,2,3$, form a pencil of metrics of constant Riemannian curvature) if and only if

$$
\left(b^{1}(u)\right)^{2}=\left(b^{2}(u)\right)^{2}=\frac{\varepsilon^{2}}{4 K}\left(u^{1}-u^{2}\right), \quad \varepsilon^{1}=-\varepsilon^{2}, \quad c= \pm \frac{1}{2} .
$$

\section{Almost compatible metrics that are not compatible}

LeMma 7.1. Two-component diagonal conformally Euclidean metric

$$
g^{i j}(u)=\exp (a(u)) \delta^{i j}, \quad 1 \leq i, j \leq 2,
$$

is flat if and only if the function $a(u)$ is harmonic, that is,

$$
\Delta a \equiv \frac{\partial^{2} a}{\partial\left(u^{1}\right)^{2}}+\frac{\partial^{2} a}{\partial\left(u^{2}\right)^{2}}=0
$$

In particular, the metric $g_{1}^{i j}(u)=\exp \left(u^{1} u^{2}\right) \delta^{i j}, 1 \leq i, j \leq 2$, is flat. It is obvious that the flat metrics $g_{1}^{i j}(u)=\exp \left(u^{1} u^{2}\right) \delta^{i j}, 1 \leq i, j \leq 2$, and $g_{2}^{i j}(u)=$ $\delta^{i j}, 1 \leq i, j \leq 2$, are almost compatible, since the corresponding Nijenhuis tensor (3.6) vanishes. But it follows from Lemma 7.1 that these metrics are not compatible and their sum is not a flat metric.

Similarly, it is also possible to construct other counterexamples to Theorem 2.5. Moreover, the following statement is true.

Proposition 7.2. Any nonconstant real harmonic function $a(u)$ defines a pair of almost compatible metrics $g_{1}^{i j}(u)=\exp (a(u)) \delta^{i j}, 1 \leq i, j \leq 2$, and $g_{2}^{i j}(u)=$ $\delta^{i j}, 1 \leq i, j \leq 2$, which are not compatible. These metrics are compatible if and only if $a=a\left(u^{1} \pm i u^{2}\right)$.

We also construct almost compatible metrics of constant Riemannian curvature that are not compatible.

LeMma 7.3. Two-component diagonal conformally Euclidean metric

$$
g^{i j}(u)=\exp (a(u)) \delta^{i j}, \quad 1 \leq i, j \leq 2,
$$


is a metric of constant Riemannian curvature $K$ if and only if the function $a(u)$ is a solution of the Liouville equation

$$
\Delta a \equiv \frac{\partial^{2} a}{\partial\left(u^{1}\right)^{2}}+\frac{\partial^{2} a}{\partial\left(u^{2}\right)^{2}}=2 K e^{-a(u)}
$$

Proposition 7.4. For the metrics $g_{1}^{i j}(u)=\exp (a(u)) \delta^{i j}, 1 \leq i, j \leq 2$, and $g_{2}^{i j}(u)=\delta^{i j}, 1 \leq i, j \leq 2$, the corresponding Nijenhuis tensor vanishes, that is, they are always almost compatible. But they are real compatible metrics of constant Riemannian curvature $K$ and 0 , respectively, only in the most trivial case when the function $a(u)$ is constant and, consequently, $K=0$. Complex metrics are compatible if and only if $a(u)=a\left(u^{1} \pm i u^{2}\right)$ and, in this case, also $K=0$.

Note that all the one-component "metrics" are always compatible, and all the one-component local Poisson structures of hydrodynamic type are also always compatible. Let us construct examples of almost compatible metrics that are not compatible for any $N>1$.

Proposition 7.5. The metrics $g_{1}^{i j}(u)=b(u) \delta^{i j}, 1 \leq i, j \leq N$, and $g_{2}^{i j}(u)=\delta^{i j}$, $1 \leq i, j \leq N$, where $b(u)$ is an arbitrary function, are always almost compatible, since the corresponding Nijenhuis tensor vanishes. But they are compatible real metrics only in the most trivial case when the function $b(u)$ is constant. Complex metrics are compatible if and only if either the function $b(u)$ is constant or $N=2$ and $b(u)=b\left(u^{1} \pm i u^{2}\right)$.

\section{Compatible flat metrics and the Zakharov method of differential reductions}

Recall the Zakharov method for integrating the Lamé equations (5.1) and (5.2) [36].

We must choose a matrix function $F_{i j}\left(s, s^{\prime}, u\right)$ and solve the following linear integral equation:

$$
K_{i j}\left(s, s^{\prime}, u\right)=F_{i j}\left(s, s^{\prime}, u\right)+\int_{s}^{\infty} \sum_{l} K_{i l}(s, q, u) F_{l j}\left(q, s^{\prime}, u\right) d q .
$$

Then, we obtain a one-parameter family of solutions of the Lamé equations by the formula

$$
\beta_{i j}(s, u)=K_{j i}(s, s, u)
$$


In particular, if $F_{i j}\left(s, s^{\prime}, u\right)=f_{i j}\left(s-u^{i}, s^{\prime}-u^{j}\right)$, where $f_{i j}(x, y)$ is an arbitrary matrix function of two variables, then formula (8.2) produces solutions of (5.1). To satisfy (5.2), Zakharov proposed to impose on the dressing matrix function $F_{i j}\left(s-u^{i}, s^{\prime}-u^{j}\right)$ a certain additional linear differential relation. If $F_{i j}\left(s-u^{i}, s^{\prime}-u^{j}\right)$ satisfy the Zakharov differential relation, then the rotation coefficients $\beta_{i j}(u)$ additionally satisfy (5.2).

We present a scheme for integrating all the system (5.1), (5.2), and (5.3).

LEMMA 8.1. If both the function $F_{i j}\left(s-u^{i}, s^{\prime}-u^{j}\right)$ and the function

$$
\tilde{F}_{i j}\left(s-u^{i}, s^{\prime}-u^{j}\right)=\frac{\sqrt{f^{j}\left(u^{j}-s^{\prime}\right)}}{\sqrt{f^{i}\left(u^{i}-s\right)}} F_{i j}\left(s-u^{i}, s^{\prime}-u^{j}\right)
$$

satisfy the Zakharov differential relation, then the corresponding rotation coefficients $\beta_{i j}(u)$ (8.2) satisfy all the equations (5.1), (5.2), and (5.3).

Proof. Actually, if $K_{i j}\left(s, s^{\prime}, u\right)$ is the solution of the linear integral equation (8.1) corresponding to the function $F_{i j}\left(s-u^{i}, s^{\prime}-u^{j}\right)$, then

$$
\tilde{K}_{i j}\left(s, s^{\prime}, u\right)=\frac{\sqrt{f^{j}\left(u^{j}-s^{\prime}\right)}}{\sqrt{f^{i}\left(u^{i}-s\right)}} K_{i j}\left(s, s^{\prime}, u\right)
$$

is the solution of (8.1) corresponding to function (8.3). It is easy to prove multiplying the integral equation (8.1) by

$$
\frac{\sqrt{f^{j}\left(u^{j}-s^{\prime}\right)}}{\sqrt{f^{i}\left(u^{i}-s\right)}}
$$

The relation

$$
\begin{aligned}
K_{i j}\left(s, s^{\prime}, u\right)= & F_{i j}\left(s-u^{i}, s^{\prime}-u^{j}\right) \\
& +\int_{s}^{\infty} \sum_{l} K_{i l}(s, q, u) F_{l j}\left(q-u^{l}, s^{\prime}-u^{j}\right) d q
\end{aligned}
$$


implies

$$
\begin{aligned}
\frac{\sqrt{f^{j}\left(u^{j}-s^{\prime}\right)}}{\sqrt{f^{i}\left(u^{i}-s\right)}} K_{i j}\left(s, s^{\prime}, u\right)= & \frac{\sqrt{f^{j}\left(u^{j}-s^{\prime}\right)}}{\sqrt{f^{i}\left(u^{i}-s\right)}} F_{i j}\left(s-u^{i}, s^{\prime}-u^{j}\right) \\
& +\int_{s}^{\infty} \sum_{l} \frac{\sqrt{f^{l}\left(u^{l}-q\right)}}{\sqrt{f^{i}\left(u^{i}-s\right)}} K_{i l}(s, q, u) \\
& \times \frac{\sqrt{f^{j}\left(u^{j}-s^{\prime}\right)}}{\sqrt{f^{l}\left(u^{l}-q\right)}} F_{l j}\left(q-u^{l}, s^{\prime}-u^{j}\right) d q
\end{aligned}
$$

and, finally, we have

$$
\begin{aligned}
\tilde{K}_{i j}\left(s, s^{\prime}, u\right)= & \widetilde{F}_{i j}\left(s-u^{i}, s^{\prime}-u^{j}\right) \\
& +\int_{s}^{\infty} \sum_{l} \widetilde{K}_{i l}(s, q, u) \widetilde{F}_{l j}\left(q-u^{l}, s^{\prime}-u^{j}\right) d q .
\end{aligned}
$$

Then both $\tilde{\beta}_{i j}(s, u)=\widetilde{K}_{j i}(s, s, u)$ and $\beta_{i j}(s, u)=K_{j i}(s, s, u)$ satisfy the Lamé equations (5.1) and (5.2). Besides, we have

$$
\begin{aligned}
\tilde{\beta}_{i j}(s, u)=\tilde{K}_{j i}(s, s, u) & =\frac{\sqrt{f^{i}\left(u^{i}-s\right)}}{\sqrt{f^{j}\left(u^{j}-s\right)}} K_{j i}(s, s, u) \\
& =\frac{\sqrt{f^{i}\left(u^{i}-s\right)}}{\sqrt{f^{j}\left(u^{j}-s\right)}} \beta_{i j}(s, u) .
\end{aligned}
$$

Thus, in this case, the rotation coefficients $\beta_{i j}(u)$ exactly satisfy all the equations (5.1), (5.2), and (5.3), that is, they generate the corresponding compatible flat metrics.

\section{Integrability of the equations for nonsingular pairs of compatible flat metrics}

The Zakharov differential reduction can be written as follows [36]:

$$
\frac{\partial F_{i j}\left(s, s^{\prime}, u\right)}{\partial s^{\prime}}+\frac{\partial F_{j i}\left(s^{\prime}, s, u\right)}{\partial s}=0
$$


Thus, to resolve these differential relations for the matrix function $F_{i j}\left(s-u^{i}, s^{\prime}-u^{j}\right)$, we can introduce $N(N-1) / 2$ arbitrary functions of two variables $\Phi_{i j}(x, y), i<j$ and put for $i<j$

$$
\begin{aligned}
& F_{i j}\left(s-u^{i}, s^{\prime}-u^{j}\right)=\frac{\partial \Phi_{i j}\left(s-u^{i}, s^{\prime}-u^{j}\right)}{\partial s}, \\
& F_{j i}\left(s-u^{i}, s^{\prime}-u^{j}\right)=-\frac{\partial \Phi_{i j}\left(s^{\prime}-u^{i}, s-u^{j}\right)}{\partial s}, \\
& F_{i i}\left(s-u^{i}, s^{\prime}-u^{i}\right)=\frac{\partial \Phi_{i i}\left(s-u^{i}, s^{\prime}-u^{i}\right)}{\partial s}, \text { for any } i,
\end{aligned}
$$

where $\Phi_{i i}(x, y), i=1, \ldots, N$, are arbitrary skew-symmetric functions of two variables:

$$
\Phi_{i i}(x, y)=-\Phi_{i i}(y, x)
$$

(see [36]).

For the function

$$
\widetilde{F}_{i j}\left(s-u^{i}, s^{\prime}-u^{j}\right)=\frac{\sqrt{f^{j}\left(u^{j}-s^{\prime}\right)}}{\sqrt{f^{i}\left(u^{i}-s\right)}} F_{i j}\left(s-u^{i}, s^{\prime}-u^{j}\right),
$$

the Zakharov differential relation (9.1) exactly gives the following $\mathrm{N}(\mathrm{N}-$ 1) / 2 linear partial differential equations of the second order for $N(N-$ 1)/2 functions $\Phi_{i j}\left(s-u^{i}, s^{\prime}-u^{j}\right), i<j$, of two variables:

$$
\begin{aligned}
& \frac{\partial}{\partial s^{\prime}}\left(\frac{\sqrt{f^{j}\left(u^{j}-s^{\prime}\right)}}{\sqrt{f^{i}\left(u^{i}-s\right)}} \frac{\partial \Phi_{i j}\left(s-u^{i}, s^{\prime}-u^{j}\right)}{\partial s}\right) \\
& \quad-\frac{\partial}{\partial s}\left(\frac{\sqrt{f^{i}\left(u^{i}-s\right)}}{\sqrt{f^{j}\left(u^{j}-s^{\prime}\right)}} \frac{\partial \Phi_{i j}\left(s-u^{i}, s^{\prime}-u^{j}\right)}{\partial s^{\prime}}\right)=0, \quad i<j,
\end{aligned}
$$

or, equivalently,

$$
\begin{gathered}
2 \frac{\partial^{2} \Phi_{i j}\left(s-u^{i}, s^{\prime}-u^{j}\right)}{\partial u^{i} \partial u^{j}}\left(f^{i}\left(u^{i}-s\right)-f^{j}\left(u^{j}-s^{\prime}\right)\right) \\
+\frac{\partial \Phi_{i j}\left(s-u^{i}, s^{\prime}-u^{j}\right)}{\partial u^{j}} \frac{d f^{i}\left(u^{i}-s\right)}{d u^{i}} \\
-\frac{\partial \Phi_{i j}\left(s-u^{i}, s^{\prime}-u^{j}\right)}{\partial u^{i}} \frac{d f^{j}\left(u^{j}-s^{\prime}\right)}{d u^{j}}=0, \quad i<j .
\end{gathered}
$$


It is very interesting that all these equations (9.6), for the functions $\Phi_{i j}\left(s-u^{i}, s^{\prime}-u^{j}\right)$, are of the same type as in the two-component case. In fact, these equations coincide with the corresponding single equation (6.3) for the two-component case.

Besides, for $N$ functions $\Phi_{i i}\left(s-u^{i}, s^{\prime}-u^{i}\right)$, we also have the following $N$ linear partial differential equations of the second order from the Zakharov differential relation (9.1):

$$
\begin{aligned}
& \frac{\partial}{\partial s^{\prime}}\left(\frac{\sqrt{f^{i}\left(u^{i}-s^{\prime}\right)}}{\sqrt{f^{i}\left(u^{i}-s\right)}} \frac{\partial \Phi_{i i}\left(s-u^{i}, s^{\prime}-u^{i}\right)}{\partial s}\right) \\
& +\frac{\partial}{\partial s}\left(\frac{\sqrt{f^{i}\left(u^{i}-s\right)}}{\sqrt{f^{i}\left(u^{i}-s^{\prime}\right)}} \frac{\partial \Phi_{i i}\left(s^{\prime}-u^{i}, s-u^{i}\right)}{\partial s^{\prime}}\right)=0
\end{aligned}
$$

or, equivalently,

$$
\begin{gathered}
2 \frac{\partial^{2} \Phi_{i i}\left(s-u^{i}, s^{\prime}-u^{i}\right)}{\partial s \partial s^{\prime}}\left(f^{i}\left(u^{i}-s\right)-f^{i}\left(u^{i}-s^{\prime}\right)\right) \\
-\frac{\partial \Phi_{i i}\left(s-u^{i}, s^{\prime}-u^{i}\right)}{\partial s} \frac{d f^{i}\left(u^{i}-s^{\prime}\right)}{d s^{\prime}} \\
+\frac{\partial \Phi_{i i}\left(s-u^{i}, s^{\prime}-u^{i}\right)}{\partial s^{\prime}} \frac{d f^{i}\left(u^{i}-s\right)}{d s}=0 .
\end{gathered}
$$

Any solution of linear partial differential equations (9.6) and (9.8) generates a one-parameter family of solutions of system (5.1), (5.2), and (5.3) by linear relations and formulas (9.2), (8.1), and (8.2). Thus, our problem is linearized.

\section{Acknowledgment}

This work was supported by the Alexander von Humboldt Foundation (Germany), the Russian Foundation for Basic Research (project no. 9901-00010), and INTAS (project no. 02-01-00803).

\section{References}

[1] M. Arik, F. Neyzi, Y. Nutku, P. J. Olver, and J. M. Verosky, Multi-Hamiltonian structure of the Born-Infeld equation, J. Math. Phys. 30 (1989), no. 6, 13381344. 
[2] D. B. Cooke, Classification results and the Darboux theorem for low-order Hamiltonian operators, J. Math. Phys. 32 (1991), no. 1, 109-119.

[3] Compatibility conditions for Hamiltonian pairs, J. Math. Phys. 32 (1991), no. 11, 3071-3076.

[4] G. Darboux, Leçons sur les Systèmes Orthogonaux et les Coordonnées Curvilignes, 2nd ed., Gauthier-Villars, Paris, 1910 (French).

[5] I. Dorfman, Dirac Structures and Integrability of Nonlinear Evolution Equations, John Wiley \& Sons, Chichester, 1993.

[6] B. Dubrovin, Geometry of 2D topological field theories, Integrable Systems and Quantum Groups (Montecatini Terme, 1993), Lecture Notes in Mathematics, vol. 1620, Springer, Berlin, 1996, pp. 120-348.

[7] Differential geometry of the space of orbits of a Coxeter group, Surveys in Differential Geometry: Integral Systems [Integrable Systems], Surveys in Differential Geometry, vol. 4, International Press, Massachusetts, 1998, pp. 181-211.

[8] Flat pencils of metrics and Frobenius manifolds, Integrable Systems and Algebraic Geometry (Kobe/Kyoto, 1997), World Scientific Publishing, New Jersey, 1998, pp. 47-72.

[9] B. Dubrovin and S. P. Novikov, Hamiltonian formalism of one-dimensional systems of the hydrodynamic type and the Bogolyubov-Whitham averaging method, Dokl. Akad. Nauk SSSR 270 (1983), no. 4, 781-785, translated in Soviet Math. Dokl. 27 (1983), 665-669.

[10] _ Hydrodynamics of weakly deformed soliton lattices. Differential geometry and Hamiltonian theory, Uspekhi Mat. Nauk 44 (1989), no. 6, 29-98, translated in Russian Math. Surveys 44 (1989), no. 6, 35-124.

[11] E. V. Ferapontov, Hamiltonian systems of hydrodynamic type and their realizations on hypersurfaces of a pseudo-Euclidean space, Problems in Geometry, Vol. 22 (Russian), Akad. Nauk SSSR Vsesoyuz. Inst. Nauchn. i Tekhn. Inform., Moscow, 1990, translated in J. Soviet Math. 55 (1991), no. 5, 1970-1995, pp. 59-96.

[12] Differential geometry of nonlocal Hamiltonian operators of hydrodynamic type, Funktsional. Anal. i Prilozhen. 25 (1991), no. 3, 37-49, translated in Funct. Anal. Appl. 25 (1991), no. 3, 195-204.

[13] Nonlocal Hamiltonian operators of hydrodynamic type: differential geometry and applications, Topics in Topology and Mathematical Physics (S. P. Novikov, ed.), Amer. Math. Soc. Transl. Ser. 2, vol. 170, American Mathematical Society, Rhode Island, 1995, pp. 33-58.

[14] E. V. Ferapontov and M. V. Pavlov, Quasiclassical limit of coupled KdV equations. Riemann invariants and multi-Hamiltonian structure, Phys. D 52 (1991), no. 2-3, 211-219.

[15] A. S. Fokas and B. Fuchssteiner, On the structure of symplectic operators and hereditary symmetries, Lett. Nuovo Cimento (2) 28 (1980), no. 8, 299-303.

[16] B. Fuchssteiner, Application of hereditary symmetries to nonlinear evolution equations, Nonlinear Anal. 3 (1979), no. 6, 849-862.

[17] C. S. Gardner, Korteweg-de Vries equation and generalizations. IV. The Kortewegde Vries equation as a Hamiltonian system, J. Mathematical Phys. 12 (1971), 1548-1551. 
[18] I. M. Gel'fand and I. Dorfman, Hamiltonian operators and algebraic structures associated with them, Funktsional. Anal. i Prilozhen. 13 (1979), no. 4, 13-30, translated in Funct. Anal. Appl. 13 (1979), 246-262.

[19] H. Gümral and Y. Nutku, Multi-Hamiltonian structure of equations of hydrodynamic type, J. Math. Phys. 31 (1990), no. 11, 2606-2611.

[20] J. Haantjes, On forming sets of eigenvectors, Indag. Math. 17 (1955), 158-162.

[21] I. M. Krichever, Algebraic-geometric n-orthogonal curvilinear coordinate systems and the solution of associativity equations, Funktsional. Anal. i Prilozhen. 31 (1997), no. 1, 32-50, translated in Funct. Anal. Appl. 31 (1997), no. 1, 25 39.

[22] F. Magri, A simple model of the integrable Hamiltonian equation, J. Math. Phys. 19 (1978), no. 5, 1156-1162.

[23] O. I. Mokhov, Local third-order Poisson brackets, Uspekhi Mat. Nauk 40 (1985), no. 5, 257-258, translated in Russian Math. Surveys 40 (1985), 233-234.

[24] Hamiltonian differential operators and contact geometry, Funktsional. Anal. i Prilozhen. 21 (1987), no. 3, 53-60, translated in Funct. Anal. Appl. 21 (1987), 217-223.

[25] - On compatible Poisson structures of hydrodynamic type, Uspekhi Mat. Nauk 52 (1997), no. 6, 171-172, translated in Russian Math. Surveys 52 (1997), no. 6, 1310-1311.

[26] _ On compatible potential deformations of Frobenius algebras and associativity equations, Uspekhi Mat. Nauk 53 (1998), no. 2, 153-154, translated in Russian Math. Surveys 53 (1998), no. 2, 396-397.

[27] _ Symplectic and Poisson structures on loop spaces of smooth manifolds, and integrable systems, Uspekhi Mat. Nauk 53 (1998), no. 3, 85-192, translated in Russian Math. Surveys 53 (1998), no. 3, 515-622.

[28] Compatible Poisson structures of hydrodynamic type and associativity equations, Tr. Mat. Inst. Steklova 225 (1999), 284-300, translated in Proc. Steklov Inst. Math. 225 (1999), no. 2, 269-284.

[29]_, Compatible Poisson structures of hydrodynamic type and the equations of associativity in two-dimensional topological field theory, Rep. Math. Phys. 43 (1999), no. 1-2, 247-256.

[30] O. I. Mokhov and E. V. Ferapontov, Nonlocal Hamiltonian operators of hydrodynamic type that are connected with metrics of constant curvature, Uspekhi Mat. Nauk 45 (1990), no. 3(273), 191-192, translated in Russian Math. Surveys 45 (1990), no. 3, 218-219.

[31] F. Neyzi, Diagonalization and Hamiltonian structures of hyperbolic systems, J. Math. Phys. 30 (1989), no. 8, 1695-1698.

[32] A. Nijenhuis, $X_{n-1}$-forming sets of eigenvectors, Indag. Math. 13 (1951), 200212.

[33] Y. Nutku, On a new class of completely integrable nonlinear wave equations. II. Multi-Hamiltonian structure, J. Math. Phys. 28 (1987), no. 11, 2579-2585.

[34] P. J. Olver, Applications of Lie Groups to Differential Equations, Graduate Texts in Mathematics, vol. 107, Springer-Verlag, New York, 1986.

[35] P. J. Olver and Y. Nutku, Hamiltonian structures for systems of hyperbolic conservation laws, J. Math. Phys. 29 (1988), no. 7, 1610-1619.

[36] V. E. Zakharov, Description of the n-orthogonal curvilinear coordinate systems and Hamiltonian integrable systems of hydrodynamic type. I. Integration of the Lamé equations, Duke Math. J. 94 (1998), no. 1, 103-139. 
[37] V. E. Zakharov and L. D. Faddeev, The Korteweg-de Vries equation is a fully integrable Hamiltonian system, Funkcional. Anal. i Priložen. 5 (1971), no. 4, 18-27, translated in Funct. Anal. Appl. 5 (1971), 280-287.

Oleg I. Mokhov: Centre for Nonlinear Studies, L.D. Landau Institute for Theoretical Physics, Russian Academy of Sciences, 2 Kosygina Street, 117940 Moscow, Russia

E-mail address: mokhov@mi.ras.ru 


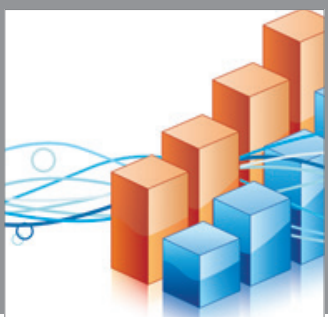

Advances in

Operations Research



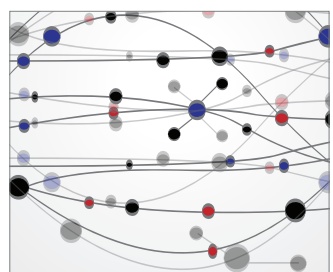

\section{The Scientific} World Journal
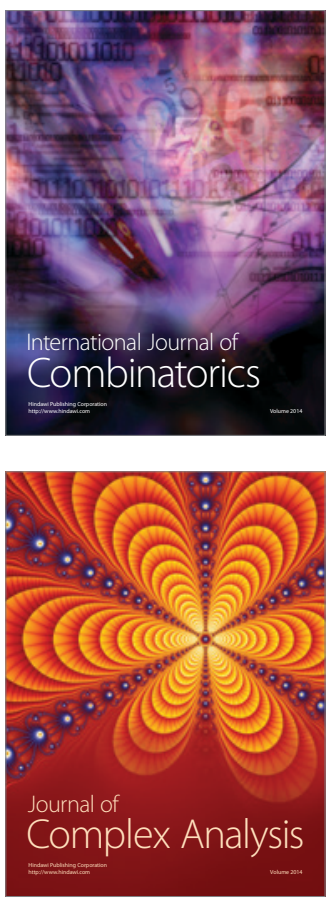

International Journal of

Mathematics and

Mathematical

Sciences


Journal of

Applied Mathematics
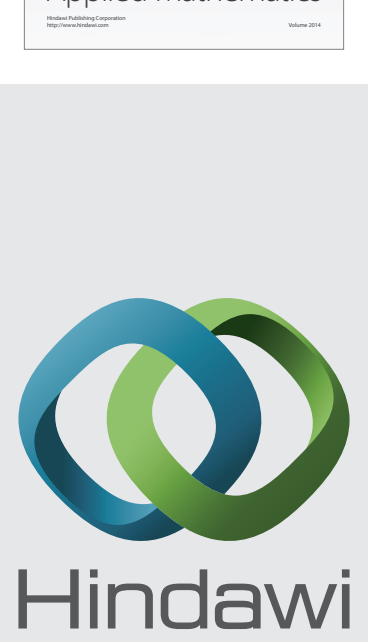

Submit your manuscripts at http://www.hindawi.com


Mathematical Problems in Engineering
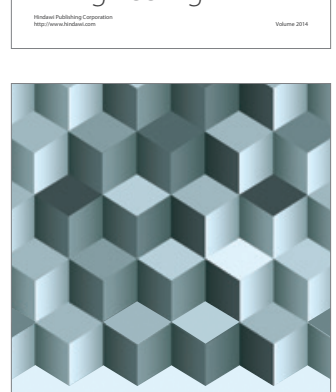

Journal of

Function Spaces


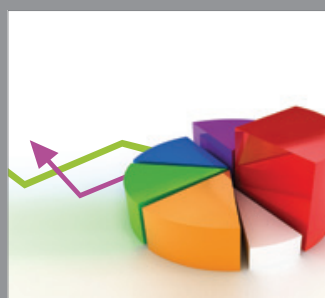

ournal of

Probability and Statistics

Promensencen
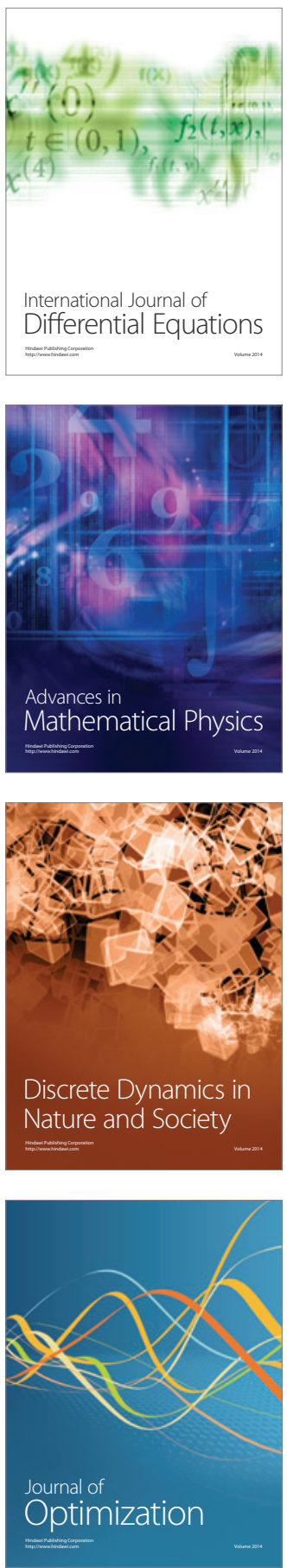